\title{
COMPORTAMIENTO SÍSMICO DE PUENTES ARCO. UN PANORAMA DEL ESTADO DEL CONOCIMIENTO
}

\author{
José de Jesús Álvarez Sereno ${ }^{(1)}$ y Ángel Carlos Aparicio Bengoechea ${ }^{(2)}$
}

\begin{abstract}
RESUMEN
Los terremotos de Loma Prieta en 1989, de Northridge en 1994, y de Hyogo-ken Nanbu en 1995, han mostrado que los puentes son estructuras sísmicamente vulnerables. Aún cuando los puentes arco no han sufrido daños severos en sismos pasados, ni aún en los eventos mencionados, esto no significa que no puedan sufrirlo en algún futuro sismo de gran intensidad. Este tipo de estructuras tiene un comportamiento complejo durante los movimientos sísmicos fuertes; ya que el arco es un elemento sometido primordialmente a una gran fuerza axial de compresión debida a la carga muerta, resulta de gran importancia conocer la magnitud de los elementos mecánicos generados durante el sismo. El objetivo de este trabajo es mostrar un panorama del estado del conocimiento que actualmente se tiene sobre la respuesta sísmica analítica de este tipo de estructuras. Los estudios referentes al análisis de la respuesta sísmica de estos puentes han sido escasos, y si ha de buscarse un proyecto óptimo es imprescindible que el diseñador cuente con información relativa a su desempeño bajo efectos sísmicos. Este trabajo es parte de los antecedentes de un proyecto de investigación que los autores están llevando a cabo, y que se describe hacia el final del documento.
\end{abstract}

\section{SUMMARY}

The 1989 Loma Prieta, 1994 Northridge and 1995 Hyogo-ken Nanbu earthquakes have proved that bridges are seismically vulnerable structures. Although arch bridges have not yet suffered damage in past earthquakes, including the above mentioned, this does not mean they will necessarily be safe in an extreme future earthquake. Arch bridge structures exhibit a complex behavior during strong earthquakes; since the arch rib is an element mainly subjected to a large axial compression force due to dead loads, it is of major importance to know how large flexural moments and axial force fluctuation are induced in the arch rib by seismic motions. A state of the art overview of the arch bridges analytical seismic response is presented in this paper, which is part of a research program that nowadays the authors are carrying out, and it is described towards the end of the document. Studies concerning the seismic response analysis of these bridges have been scarce, and for getting an optimal project it is imperative that the designer counts on information with regard to their seismic performance.

Artículo recibido el 15 de febrero de 2002 y aprobado para su publicación el 14 de febrero de 2003. Se aceptarán comentarios y/o discusiones hasta cinco meses después de su publicación.

(1) Facultad de Ingeniería Civil, Universidad Michoacana de San Nicolás de Hidalgo, Av. Lázaro Cárdenas No. 1651 P. B., 58260 Morelia, Mich., asereno@zeus.umich.mx

(2) Departamento de Ingeniería de la Construcción, Universidad Politécnica de Cataluña, angel.carlos.aparicio@upc.es 


\section{ANTECEDENTES Y ALCANCE}

Desde la aparición del presforzado, que posibilita la construcción de puentes rectos de gran claro y de puentes atirantados, los puentes arco, en particular los de concreto, experimentaron durante varios años un estancamiento prolongado. La utilización de grandes cimbras constituía la dificultad más importante que presentaba la construcción de estas estructuras, ubicadas generalmente en zonas de difícil acceso, grandes valles o cursos de agua importantes (Manterola, 1999).

Sin embargo, la aplicación a los arcos del método de construcción por avance en voladizo ha hecho resurgir esta tipología estructural a nivel mundial, en un intervalo de claros que oscila entre los 100 y los $400 \mathrm{~m}$ para el caso del concreto, o hasta los $500 \mathrm{~m}$ en el caso del acero. Los actuales récords mundiales son el Puente Wanxian (China), con un claro principal de $420 \mathrm{~m}$, y el Puente New River Gorge (E.U.A.), con un claro principal de $518.5 \mathrm{~m}$, para puentes de concreto y de acero, respectivamente, aunque se han llegado a proyectar arcos de hasta $600 \mathrm{~m}$ de claro (Spielmann, 2001).

Así pues, los puentes arco representan ya uno de los tres tipos de puentes de gran claro, junto con los atirantados y los colgantes. El arco es un elemento sometido primordialmente a compresión bajo cargas permanentes; para esto, la directriz del arco debe seguir la curva antifunicular de dichas cargas. Naturalmente, las acciones sísmicas generan condiciones cambiantes de los elementos mecánicos, rotaciones, fluencia de los apoyos y efectos adicionales de pandeo dentro o fuera del plano. La presencia de la compresión en el arco mantienen latentes los problemas de pandeo y si ésta cambia modifica las cargas críticas de diseño.

Aún cuando los puentes arco no han sufrido daño severo en el pasado, esto no significa que no puedan sufrirlo en algún futuro sismo de gran magnitud; dado que los puentes arco se construyen generalmente en sitios rocosos, hasta ahora no han experimentado movimientos sísmicos violentos. Sin embargo, existen puentes arco que han quedado sísmicamente deficientes ante los códigos actuales de diseño y que han sido motivo de estudio, como el Puente Bixby Creek en la costa de California (McCallen et al., 1999), y como los Puentes Krk I y II, construidos en 1980 en Croacia (Simunic et al., 2001); vale la pena recordar que el Puente Krk I fue record mundial de los puentes arco de concreto con sus $390 \mathrm{~m}$ de claro principal.

En años recientes, algunos investigadores han profundizado en el estudio de la respuesta sísmica de puentes arco; sin embargo, los estudios relativos al tema son escasos. La investigación bibliográfica que se presenta a continuación se divide en puentes de acero y puentes de concreto; quedan fuera del alcance de este trabajo puentes construidos con otros materiales. En los casos descritos se presentan los resultados con alcances diversos, de acuerdo al interés particular de cada uno de los autores consultados. Hacia el final del documento se presenta una discusión, donde se pretende analizar la interacción de las variables que intervienen en el comportamiento de los puentes arco durante la ocurrencia de un terremoto. El trabajo termina con algunos comentarios sobre las perspectivas de investigación, entre las que destacan la necesidad de profundizar en el estudio de la variabilidad espacial, los efectos de fuente cercana, la interacción fuerza axial - momento flexionante y la respuesta empleando sistemas de control. 


\section{PUENTES DE ACERO}

\section{Puentes sometidos a aceleración longitudinal}

Una de las investigaciones pioneras que han permitido entender el comportamiento sísmico de puentes arco con tablero superior se debe a Kuranishi y Nakajima (1986), quienes estudiaron las características dinámicas fundamentales y el comportamiento hasta la falla de arcos biarticulados y puentes arco de acero en dos dimensiones sometidos a excitaciones longitudinales armónica y sísmica, considerando la fluencia del material.

En la fig. 1 se muestran las configuraciones de los arcos parabólicos biarticulados y de los puentes estudiados. El claro principal se consideró igual a $100 \mathrm{~m}$, con una flecha de $15 \mathrm{~m}$, para una relación $\mathrm{f} / \mathrm{L}=1 / 6.67$. La relación de esbeltez de $\operatorname{los} \operatorname{arcos} \lambda_{\mathrm{A}}$ se definió como el cociente de la longitud del eje del arco $\mathrm{L}_{\mathrm{S}} \mathrm{y}$ el radio de giro de la sección transversal. La relación de rigideces $\tau$ se definió como el cociente del momento de inercia de la sección transversal del arco y el del tablero. Se estudiaron relaciones de esbeltez de 150, 200 y 300, y relaciones $\tau$ de $0.5,1.0$ y 2.0 , valores frecuentes en la práctica del diseño de la época; adicionalmente se consideraron los valor de $\tau$ iguales a 1000 e $\infty$.

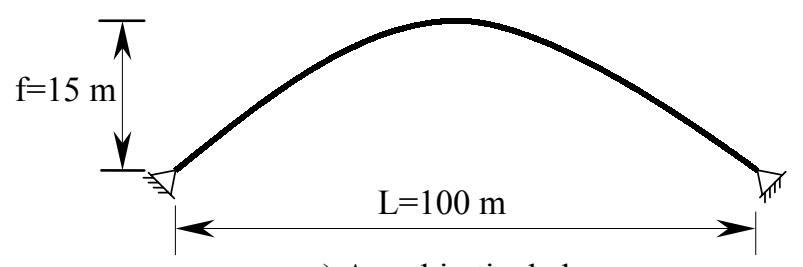

a) Arco biarticulado

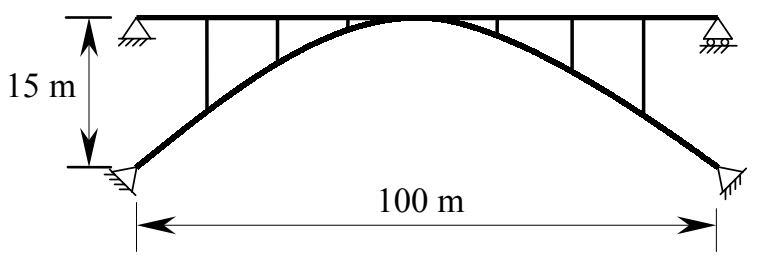

b) Puente con conexión rígida tablero-clave del arco

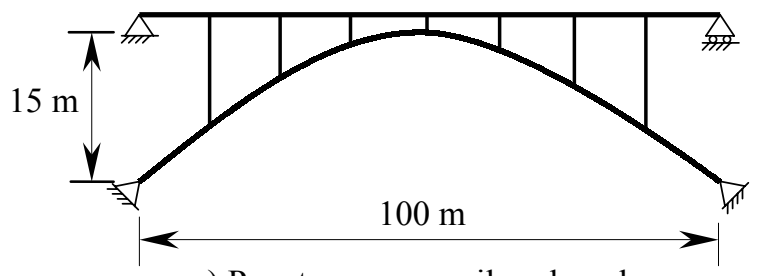

c) Puente arco con pila sobre clave

Figura 1. Configuraciones de los modelos analíticos de Kuranishi y Nakajima (1986) 
Las excitaciones aplicadas simultáneamente a los apoyos consistieron en aceleraciones longitudinales senoidales con periodo igual al primer modo de vibrar de las estructuras. Para el acero se consideró un comportamiento elasto-plástico perfecto y no se tomaron en cuenta los esfuerzos residuales. Se supuso nulo el amortiguamiento estructural. La carga muerta se consideró igual al 50\% de la carga estática última.

Un resumen de los resultados obtenidos se presenta en la tabla 1, donde $\lambda_{\mathrm{A}}$ y $\tau$ se definieron con anterioridad, y $Z$ representa la amplitud de la aceleración senoidal $\left(\mathrm{en} \mathrm{m} / \mathrm{s}^{2}\right.$ ) para la que las estructuras se colapsaron al cabo de diez ciclos de aplicación del movimiento; las condiciones de apoyo del tablero R-R, A-A y R-A, así como la longitud $\mathrm{L}_{\mathrm{PC}}$ y $\mathrm{T}_{1}$ se definen en la misma tabla.

Una primera tendencia en el comportamiento de los arcos se observó para los modelos A, B y G. El proceso de colapso, que consistió en la formación de dos articulaciones plásticas a $1 / 4$ y a $3 / 4$ del claro, no se vio afectado por la relación de esbeltez, pero la resistencia disminuyó al aumentar dicha relación.

Para los puentes arco biarticulados con una relación de esbeltez de 200 y tablero apoyado sobre rodillos, se estudió la influencia de la relación de rigideces $\tau$, casos B, C, D, E y F. Como se aprecia en la última columna de la tabla 1, la amplitud de la aceleración de colapso aumentó considerablemente al incrementar la inercia del tablero, con el consecuente decremento de $\tau$. El proceso de colapso fue similar al descrito en el párrafo anterior para los arcos.

\begin{tabular}{|c|c|c|c|c|c|c|}
\hline Modelo & $\lambda_{\mathrm{A}}$ & $\tau$ & $\begin{array}{c}\text { Condición } \\
\text { de apoyo }\end{array}$ & $\mathbf{L}_{\mathbf{P C}}$ & $\mathbf{T}_{1}$ & $\mathbf{Z}$ \\
\hline A & 150 & $\infty$ & - & - & 2.405 & 0.4 \\
\hline B & 200 & $\infty$ & - & - & 3.298 & 0.3 \\
\hline $\mathrm{C}$ & 200 & 1000 & R-R & - & 3.334 & 0.5 \\
\hline $\mathrm{D}$ & 200 & 2.0 & R-R & - & 2.199 & 1.5 \\
\hline$E$ & 200 & 1.0 & R-R & - & 1.866 & 2.0 \\
\hline $\mathrm{F}$ & 200 & 0.5 & R-R & - & 1.543 & 3.0 \\
\hline $\mathrm{G}$ & 300 & $\infty$ & - & - & 3.803 & 0.3 \\
\hline $\mathrm{H}$ & 300 & 1.0 & R-R & - & 3.135 & 1.0 \\
\hline I & 300 & 1.0 & R-A & - & 1.231 & $>10.0$ \\
\hline $\mathrm{J}$ & 300 & 1.0 & A-A & - & 1.204 & $>10.0$ \\
\hline $\mathrm{K}$ & 300 & 1.0 & R-R & 2.0 & 2.836 & 1.0 \\
\hline $\mathrm{L}$ & 300 & 1.0 & R-A & 2.0 & 1.334 & $>10.0$ \\
\hline $\mathrm{M}$ & 300 & 1.0 & R-R & 10.0 & 2.850 & $>5.0$ \\
\hline \multicolumn{3}{|c|}{$\begin{array}{l}\text { R-R: Apoyo rodillo-rodillo } \\
\text { A-A: Apoyo articulación-articulación } \\
\text { R-A: Apoyo rodillo-articulación }\end{array}$} & \multicolumn{4}{|c|}{$\begin{array}{l}\mathrm{L}_{\mathrm{PC}} \text { : Longitud de la pila central, en } \mathrm{m} \\
\mathrm{T}_{1} \text { : Periodo del primer modo longitudinal, en } \mathrm{s} \\
\mathrm{Z} \text { : Amplitud de la aceleración senoidal de falla, } \mathrm{m} / \mathrm{s}^{2}\end{array}$} \\
\hline
\end{tabular}


Se analizaron tres condiciones de apoyo para el tablero y conexión rígida arco-tablero (modelos H, I y J, con $\lambda_{\mathrm{A}}$ igual a 300): rodillo-rodillo (tipo R-R), rodillo-articulación (tipo R-A) y articulación-articulación (A-A). Dadas estas condiciones, la resistencia última ante carga vertical del modelo H (tipo R-R) resultó menor y el periodo del modo fundamental fue más largo que el de los otros dos casos; entre los modelo I y J no hubo diferencias entre la capacidad última a carga vertical y el primer modo de vibrar. Naturalmente, hubo diferencias significativas en el comportamiento; el modelo $H$ colapsó para una amplitud relativamente pequeña $\left(Z=1.0 \mathrm{~m} / \mathrm{s}^{2}\right)$, mientras que los otros dos modelos no colapsaron aun para $Z=10 \mathrm{~m} / \mathrm{s}^{2}$.

En la fig. 2 se muestran las zonas de fluencia antes del colapso para el modelo $\mathrm{H}$ y en el último ciclo para el modelo I. Para el modelo $\mathrm{H}$ se formaron dos articulaciones plásticas en el arco, así como zonas de fluencia esparcidas en el tablero y en las pilas. Para el modelo I se plastificó la zona próxima al apoyo articulado fijo del tablero, aunque el arco no colapsó. Para el modelo J no ocurrió fluencia en ninguna sección transversal; esta condición de apoyo del tablero (A-A) proporcionó al modelo la mayor resistencia dinámica. Dicha condición no podría adoptarse en un caso práctico ya que con ella serían de esperarse importantes esfuerzos debidos a variaciones térmicas, a menos que se empleasen conectores sísmicos o amortiguadores viscosos (Boitel y De La Fuente, 1998), ya que éstos permiten la contracción / dilatación del tablero ante solicitaciones reológicas y térmicas, y conectan rígidamente la estructura a los apoyos cuando ésta se somete a cargas sísmicas.

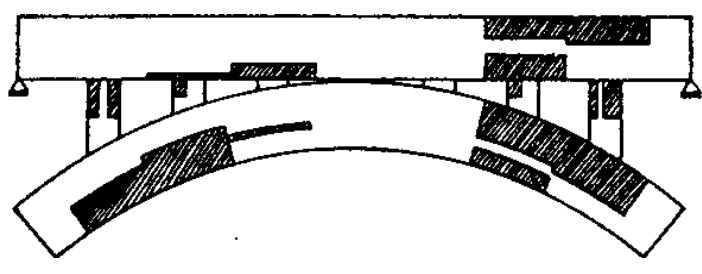

a) Tablero apoyado sobre rodillo-rodillo

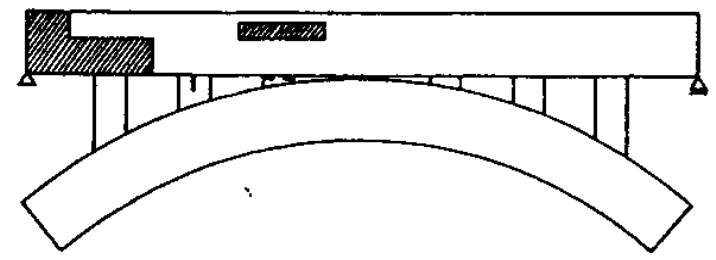

b) Tablero apoyado sobre articulación-rodillo

Figura 2. Zonas de fluencia en los puentes con conexión rígida tablero-arco (escaneada de Kuranishi y Nakajima, 1986)

No se detectaron diferencias significativas entre el modelo $\mathrm{H}$ (con conexión rígida arcotablero y tablero apoyado sobre rodillos) y el K (con pila central de $2 \mathrm{~m}$ de longitud), y entre el modelo I y el L, aunque las secciones transversales de las pilas de los modelos con pila central fluyeron ampliamente cuando el tablero era tipo R-A.

\section{Respuesta de tres puentes con tablero superior}

Dusseau y Wen (1989) estudiaron tres puentes existentes con claros principales de 59, 213 y 518 m. Estos claros corresponden a los puentes South Street Bridge (SSB), Cold Spring Canyon Bridge (CSCB) y New River Gorge Bridge (NRGB), respectivamente. En la fig. 3 se muestra la geometría en elevación de los tres modelos tridimensionales. Aunque los tres son puentes con 
tablero superior, tienen diferencias importantes en lo que se refiere a juntas de dilatación, y transmisión de fuerzas lateral y longitudinal entre el tablero y el arco.

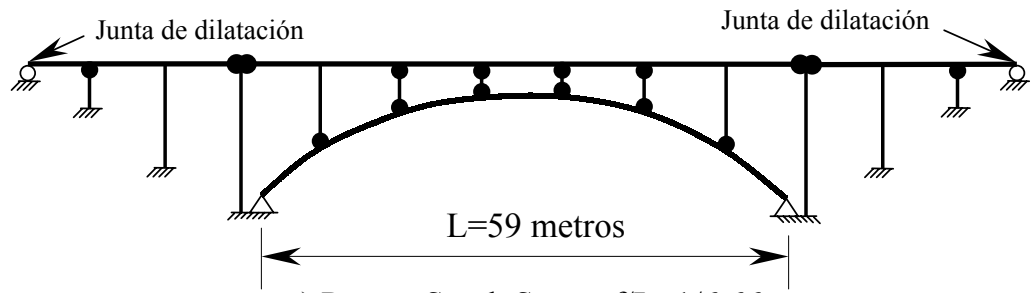

a) Puente South Street, $f / L=1 / 6.66$

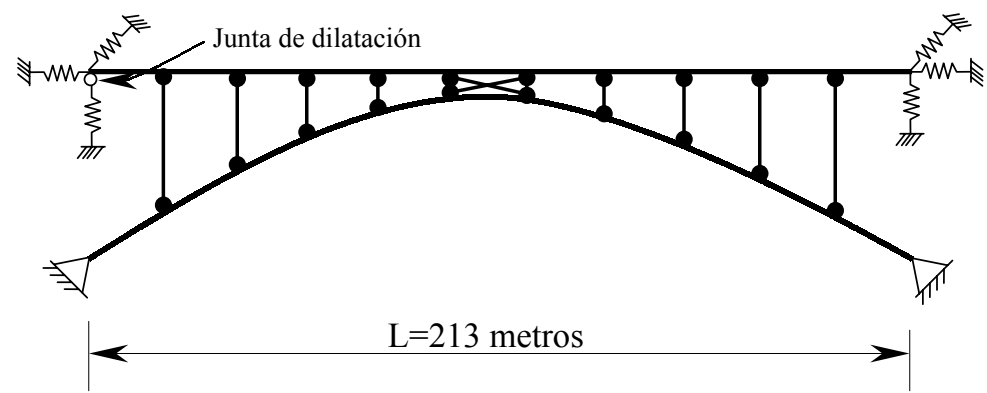

b) Puente Cold Spring Canyon, $\mathrm{f} / \mathrm{L}=1 / 6$

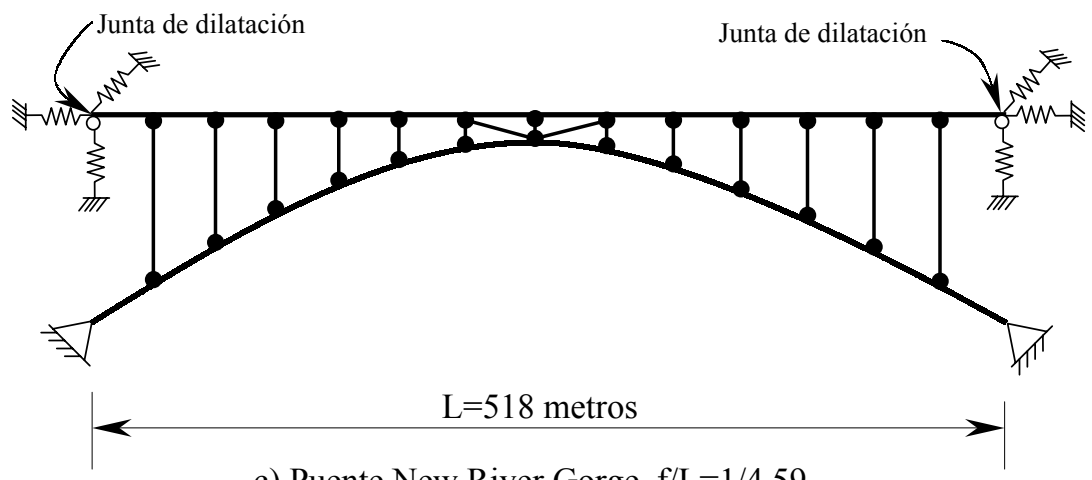

c) Puente New River Gorge, $\mathrm{f} / \mathrm{L}=1 / 4.59$

Figura 3. Modelos analíticos de tres puentes arco de acero (Dusseau y Wen, 1989)

Uno de los resultados que llaman la atención es que la relación periodo natural a claro del arco resultó muy similar para los tres puentes, a pesar de sus diferencias en longitud, sistema constructivo y localización. En la tabla 2 se presentan las relaciones periodo/claro (segundos/metro) para la respuesta en el plano (desplazamientos verticales) y fuera del plano para los primeros cuatro modos de vibrar en cada dirección. Los modos considerados en el plano correspondían a los relacionados con movimientos verticales; los modos correspondientes al movimiento longitudinal no fueron considerados aquí puesto que estos dependían de la configuración estructural en esta dirección, que era muy diferente para cada puente.

Para el modelo SSB se realizó un análisis sísmico modal espectral combinando las respuestas modales mediante el método de la raíz cuadrada de la suma de los cuadrados, mientras 
que para los modelos CSCB y NRGB se hizo un análisis paso a paso, con la finalidad de estudiar el efecto de la variabilidad espacial del movimiento sísmico en los apoyos. Se emplearon acelerogramas artificiales separadamente en tres direcciones ortogonales, considerando tres niveles de aceleración: $0.09 g, 0.22 g$ y $0.50 g$. Para explorar el efecto de la variabilidad espacial, sólo se consideró el movimiento no sincrónico de los apoyos con la finalidad de tener una medida de la respuesta de estos puentes ante tal fenómeno.

\begin{tabular}{|c|c|c|c|c|c|c|c|c|}
\hline \multicolumn{1}{|c|}{ Tabla 2. Relación periodo natural/claro del arco (s/m), (Dusseau y Wen, 1989) } \\
\hline Movimiento: & \multicolumn{3}{c|}{ En el plano } & \multicolumn{4}{c|}{ Fuera del plano } \\
\hline Modo: & 1 & 2 & 3 & 4 & 1 & 2 & 3 & 4 \\
\hline SSB & 0.01051 & 0.00593 & 0.00322 & 0.00305 & 0.01034 & 0.00661 & 0.00458 & 0.00254 \\
\hline CSCB & 0.01089 & 0.00559 & 0.00296 & 0.00202 & 0.01277 & 0.00789 & 0.00531 & 0.00296 \\
\hline NRGB & 0.00807 & 0.00386 & 0.00276 & 0.00203 & 0.01309 & 0.00672 & 0.00463 & 0.00365 \\
\hline Promedio & 0.00982 & 0.00513 & 0.00298 & 0.00237 & 0.01207 & 0.00707 & 0.00484 & 0.00305 \\
\hline
\end{tabular}

Los resultados mostraron que en ninguno de los casos considerados los esfuerzos totales en los miembros principales excedieron el esfuerzo de fluencia del acero. La respuesta ante movimiento transversal uniforme fue, generalmente, la más desfavorable, mientras que la respuesta al movimiento vertical fue menor que la debida al movimiento transversal $o$ longitudinal.

Para los dos puentes con claros mayores sometidos a excitación longitudinal uniforme, los esfuerzos en el arco debidos a sismo fueron menores que los debidos a carga muerta, aun para $0.50 \mathrm{~g}$. Sin embargo, los esfuerzos en el tablero debidos a sismo longitudinal excedieron los de carga muerta para $0.50 \mathrm{~g}$. Los esfuerzos debidos al movimiento vertical excedieron los debidos a carga viva para $0.22 \mathrm{~g}$. En resumen, el esfuerzo máximo al que se vieron sometidos los arcos fue igual al 78\% del esfuerzo de fluencia para sismo transversal (modelo CSCB para $0.50 \mathrm{~g}$ ) y para los tableros fue igual al 93\% del esfuerzo de fluencia para sismo longitudinal (modelo NRGB para $0.50 g)$.

La respuesta en miembros secundarios dependió fuertemente de los detalles del diseño. Los mayores desplazamientos longitudinales ocurrieron para el modelo SSB, en el que se alcanzó el esfuerzo de fluencia en las columnas para $0.09 \mathrm{~g}$. Los arriostramientos que sujetaban el tablero y el arco para el modelo CSCB podrían alcanzar su resistencia a la rotura si el movimiento lateral llegase a $0.22 \mathrm{~g}$. Para el modelo NRGB, los miembros secundarios podrían alcanzar el esfuerzo de fluencia si la aceleración longitudinal del terreno alcanzase $0.50 \mathrm{~g}$.

Los efectos del movimiento sísmico no sincrónico en los apoyos fueron menores en las direcciones vertical y transversal, comparados con los debidos a movimiento uniforme, mientras que ocurrió lo contrario para el movimiento longitudinal. Uno de los casos más desfavorable en dirección longitudinal consistió en aplicar primeramente un cierto acelerograma a los apoyos de un extremo del puente, dejando transcurrir un lapso de tiempo de 0.25 segundos para el modelo CSCB y de 0.60 segundos para el NRGB, basándose en una velocidad de propagación de las ondas sísmicas en un medio rocoso de $850 \mathrm{~m} / \mathrm{s}$, y transcurrido este tiempo aplicarlo a los apoyos del otro extremo del puente. Bajo esta condición de carga, el modelo NRGB exhibió un 12\% de 
incremento en el esfuerzo máximo total en el arco comparado con la respuesta ante movimiento uniforme, mientras que el CSCB mostró un $84 \%$ de incremento.

Para mejorar estos análisis sería necesario considerar la acción tridimensional del sismo, contar con acelerogramas realistas que permitiesen evaluar el efecto de la variabilidad espacial y considerar la influencia de la no linealidad geométrica, ya que ésta tiende a incrementar la respuesta (Lee, 1990). Además, se consideró un amortiguamiento del 5\%, valor que parece alto para estructuras de acero; al bajarlo se incrementaría la respuesta.

\section{Análisis sísmico no lineal de tres puentes}

En la Universidad Estatal de Michigan, Lee (1990) desarrolló un método para el análisis sísmico no lineal de puentes arco, el cual toma en cuenta la no linealidad del material o la geométrica, para las barras de eje curvo que conforman el (los) arco(s). Elaboró un programa de computadora que permitía realizar un análisis paso a paso. Estudió los mismos puentes considerados por Dusseau y Wen (1989), descritos con anterioridad: el South Street Bridge (SSB), el Cold Spring Canyon Bridge (CSCB) y el New River Gorge Bridge (NRGB), con claros principales de 59, 213 y $518 \mathrm{~m}$ (fig. 3).

Para el análisis se elaboraron modelos tridimensionales, aplicando un acelerograma en tres direcciones ortogonales. Se trató de un acelerograma artificial que intentaba representar el movimiento cercano al epicentro para un sismo de magnitud 8 , con una aceleración máxima del terreno de $0.39 \mathrm{~g}$, y una duración de 120 segundos; para los diferentes análisis se emplearon los primeros 45 segundos del acelerograma. La aceleración vertical se consideró igual al 75\% de la horizontal. Se aplicó simultáneamente el mismo movimiento del suelo a todos los apoyos.

De las historias temporales (desplazamiento-tiempo o fuerza interna-tiempo) se pudo observar que aun para los casos no lineales las respuestas generalmente estaban dominadas por los modos fundamentales (en el plano del puente o fuera de éste), excepto en aquellos puntos donde la respuesta del modo fundamental era pequeña. Una historia de desplazamientos típica se presenta en la fig. 4 para la clave del arco del modelo CSCB (fig. 3.b); en este caso el periodo dominante de la respuesta coincidió con el del primer modo de vibrar (3 segundos).

Para los casos en los que se consideró la no linealidad del material, las fuerzas internas en el (los) arco(s) estuvieron limitadas por la fluencia del acero, por lo que si el proyectista permitiese el daño debido a deformaciones plásticas, las fuerzas de diseño podrían reducirse en comparación con las obtenidas mediante un análisis elástico, con el consecuente ahorro de material. En la tabla 3 se presentan los valores máximos de las fuerzas internas para una sección del arco del modelo CSCB (fig. 3.b) situada a 1/8 del claro, donde se aprecia la diferencia entre las respuestas elástica e inelástica; la respuesta inelástica estuvo limitada por las fuerzas de plastificación total.

Para los casos de no linealidad geométrica, en general, la mayor influencia del comportamiento se presentó en el periodo dominante de las estructuras, el cual se vio 
incrementado entre un 5\% y un $10 \%$ comparado con la solución lineal, ya que la fuerza axial debida a carga muerta que actuaba en los arcos redujo la rigidez de éstos.

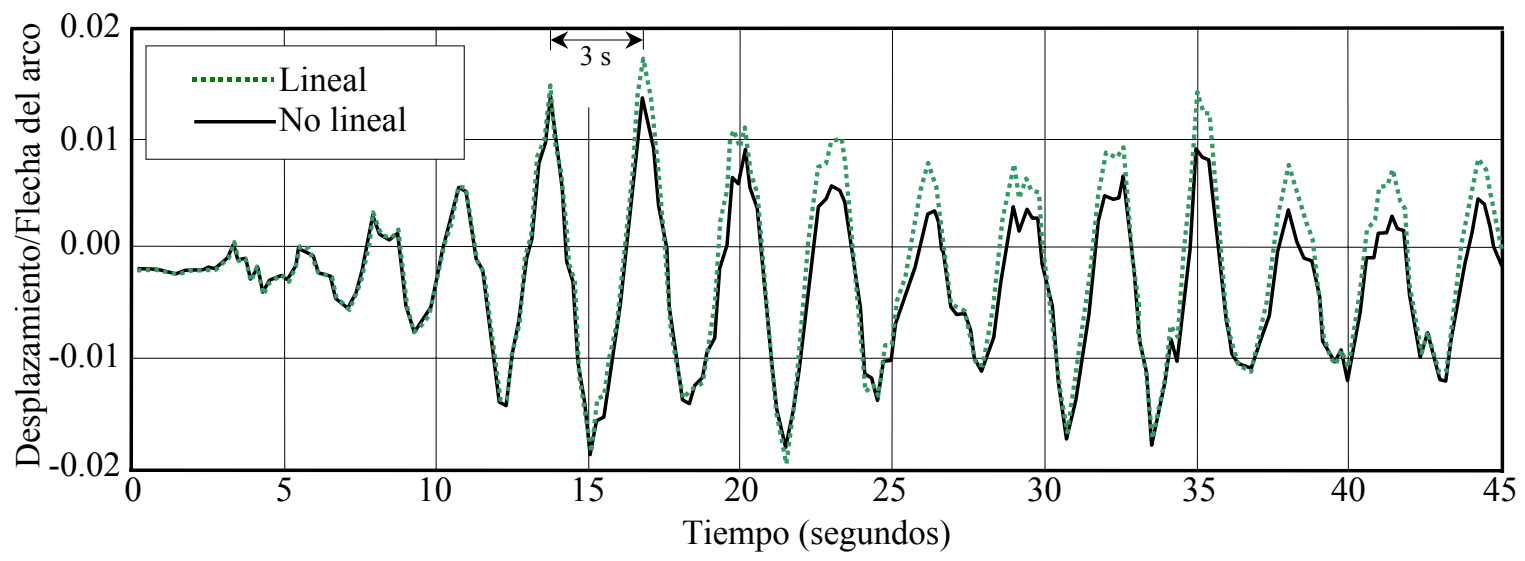

Figura 4. Historia de desplazamientos verticales para la clave del arco del modelo CSCB (Lee, 1990)

Es importante señalar que las historias temporales de disipación de energía indicaron que la energía disipada por amortiguamiento para un valor de éste del 1.5\% fue mayor que el 70\% del total (compuesta además por la energía elástica de deformación, la energía plástica y la cinética) para todos los modelos. Para un factor de amortiguamiento del 5\%, la energía disipada por amortiguamiento alcanzó el $96 \%$ del total. Estas observaciones enfatizan la importancia del amortiguamiento en la respuesta.

Tabla 3. Valores máximos de las fuerzas internas para una sección del arco del modelo CSCB situada a 1/8 del claro (Lee, 1990)

\begin{tabular}{|c|c|c|c|c|}
\hline $\begin{array}{c}\text { Fuerza } \\
\text { interna }\end{array}$ & Lineal & No lineal & $\begin{array}{c}\text { Reducción } \\
(\mathbf{\%})\end{array}$ & $\begin{array}{c}\text { Fuerza de } \\
\text { plastificación }\end{array}$ \\
\hline $\mathrm{P}_{\mathrm{z}}(\mathrm{MN})$ & 40.43 & 35.10 & 13.2 & 44.78 \\
\hline $\mathrm{M}_{\mathrm{y}}(\mathrm{MN} \cdot \mathrm{m})$ & 59.47 & 39.79 & 33.1 & 40.73 \\
\hline $\mathrm{M}_{\mathrm{z}}(\mathrm{MN} \cdot \mathrm{m})$ & 5.94 & 5.53 & 6.9 & 16.50 \\
\hline
\end{tabular}

\section{Comportamiento sísmico de dos puentes con tablero superior}

El comportamiento sísmico tridimensional no lineal de dos puentes arco fue investigado por Nazmy y Konidaris (1994). En el análisis fueron consideradas la no linealidad debida al cambio de geometría producido por los desplazamientos y la interacción fuerza axial-momento flexionante. Se consideraron excitaciones uniforme y múltiple de los apoyos. Los dos objetivos principales de esta investigación fueron: i) determinar si había una diferencia considerable entre el comportamiento lineal y no lineal empleando modelos tridimensionales y examinar el efecto del claro en la no linealidad, y ii) estudiar la diferencia en la respuesta no lineal ante excitación uniforme y múltiple en los apoyos, para evaluar el efecto de la variabilidad espacial. 


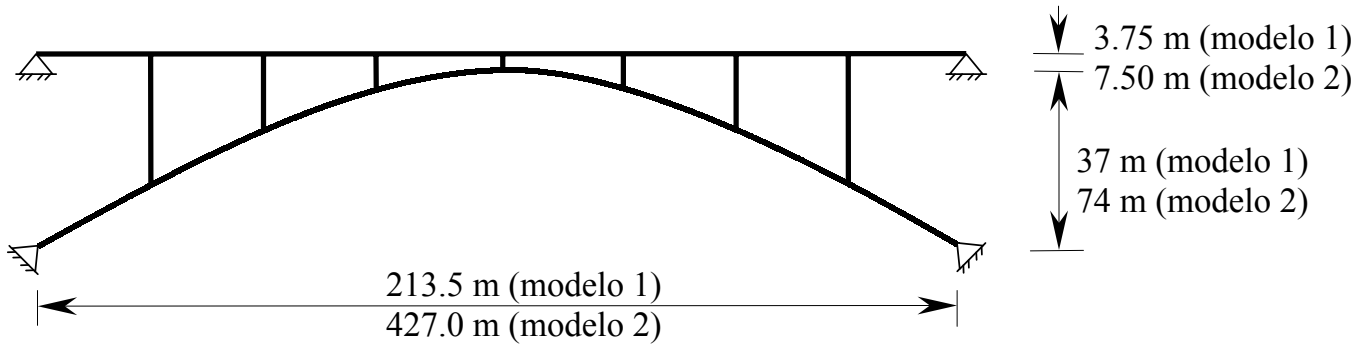

Figura 5. Modelos analíticos de Nazmy y Konidaris (1994)

Se estudiaron dos modelos 3-D de puentes arco, uno de los cuales tenía el doble de longitud que el otro, con la finalidad de investigar el efecto de la longitud del claro en las características dinámicas y en la respuesta sísmica no lineal de este tipo de estructuras. La fig. 5 muestra la geometría en elevación de ambos modelos; el modelo 1 tuvo un claro de $213.5 \mathrm{~m}$, mientras que para el modelo 2 fue de $427 \mathrm{~m}$, con una relación f/L=1/5.77.
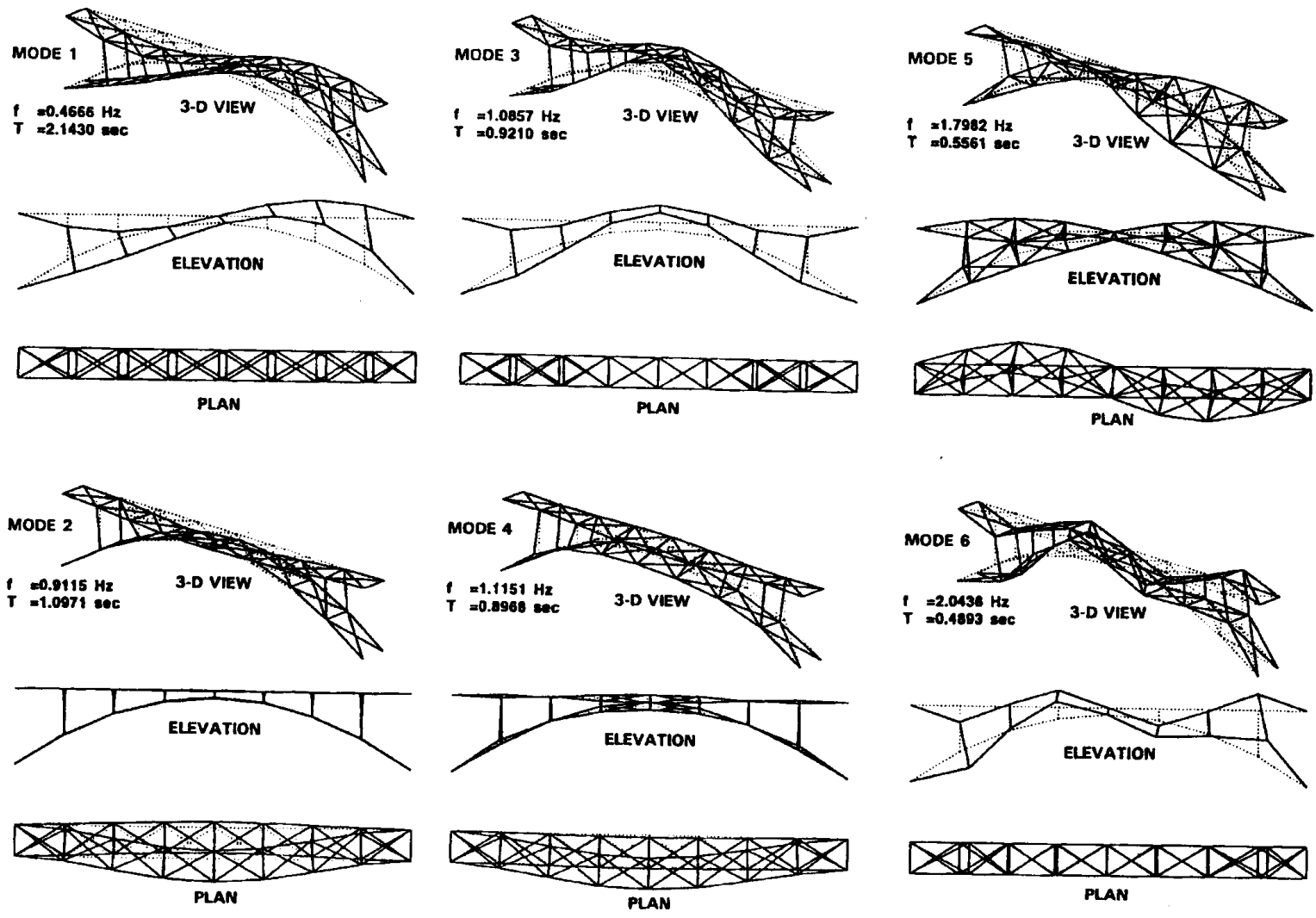

Figura 6. Modos de vibrar para el modelo 1 (escaneada de Nazmy y Konidaris, 1994)

En la fig. 6 se presentan los seis primeros modos de vibrar calculados para el modelo 1. Resulta evidente que existió un fuerte acoplamiento entre los movimientos laterales y torsionales de los arcos dentro de varios modos de vibrar; las deformaciones involucradas no pueden 
captarse en un análisis en dos dimensiones, por lo que para este tipo de estructuras se requiere un análisis tridimensional.

Debido a la magnitud de la fuerza axial en el arco ocasionada por la carga muerta, su rigidez se vio disminuida, modificando las propiedades dinámicas de la estructura. En la tabla 4 se presentan los periodos naturales para los seis primeros modos del modelo 2, obtenidos mediante un análisis lineal y a través de un análisis no lineal, considerando la degradación de rigidez del arco debida a la fuerza axial producida por la carga muerta. Un incremento considerable en los periodos (más del 46\% para el primer modo) se observa en la tabla.

Para estudiar el comportamiento sísmico se seleccionaron dos acelerogramas del sismo de El Centro de 1979. Se consideraron dos casos de tres componentes ortogonales del sismo actuando simultáneamente en los apoyos: i) excitación de entrada múltiple actuando dos señales diferentes a uno y otro lado de los modelos, y ii) excitación uniforme, con la señal más intensa de las dos anteriores actuando en ambos lados de los modelos.

\begin{tabular}{|c|c|c|c|}
\hline \multicolumn{2}{|c|}{ Tabla 4. Periodos naturales del modelo 2 (Nazmy y Konidaris, 1994) } \\
\hline Modo No. & $\begin{array}{c}\text { Periodo, análisis lineal } \\
\text { por carga muerta (s) }\end{array}$ & $\begin{array}{c}\text { Periodo, análisis no lineal } \\
\text { por carga muerta (s) }\end{array}$ & $\begin{array}{c}\text { Incremento de } \\
\text { periodo (\%) }\end{array}$ \\
\hline 1 & 5.26 & 7.69 & 46.2 \\
\hline 2 & 2.63 & 3.23 & 22.8 \\
\hline 3 & 2.44 & 3.13 & 28.3 \\
\hline 4 & 2.13 & 2.44 & 14.6 \\
\hline 5 & 1.33 & 1.52 & 14.3 \\
\hline 6 & 1.23 & 1.32 & 7.3 \\
\hline
\end{tabular}

Se efectuaron dos tipos de análisis para ambos modelos: análisis lineal de la respuesta sísmica usando superposición modal con 20 modos y análisis no lineal por carga muerta, y análisis sísmico no lineal (combinado también con análisis no lineal por carga permanente) usando el método $\theta$ de Wilson.

Los resultados no mostraron una diferencia significativa en la respuesta dinámica lineal y no lineal para el modelo de $213.5 \mathrm{~m}$ de claro y excitación uniforme de los apoyos, en el que el valor absoluto del desplazamiento vertical máximo de la clave y la fuerza axial en los riñones del $\operatorname{arco}(\mathrm{a} 1 / 4$ y a $3 / 4$ del claro) fueron similares para ambos análisis (lineal y no lineal). Por el contrario, una reducción considerable de la respuesta máxima se observó para el modelo 2 con $427 \mathrm{~m}$ de claro, cuando se realizó el análisis dinámico no lineal. Se observó una reducción de casi el $65 \%$ del desplazamiento vertical de la clave del arco, y del 58\% de la fuerza axial en los riñones del arco. Estos resultados muestran que existe un efecto importante de la no linealidad en el comportamiento sísmico de estas estructuras, lo que implica la necesidad de realizar análisis no lineales tanto por carga vertical como por sismo en puentes arco de gran claro (del orden de 300 m en adelante, según Nazmy y Konidaris).

Conforme crece la longitud de un puente, la suposición de que los apoyos son excitados uniformemente por el movimiento sísmico parece poco realista, por lo que para el modelo 2 de 
427 m de claro se comparó su respuesta ante excitación uniforme y múltiple. Esta comparación mostró que la excitación múltiple incrementó la respuesta sísmica al excitar más modos de vibrar debido al movimiento fuera de fase de los apoyos; por ejemplo, se duplicó la fuerza axial en los riñones del arco para el caso de excitación múltiple.

En cuanto a las condiciones de apoyo se refiere, ambos extremos del tablero de los modelos de la fig. 5 se supusieron apoyados sobre articulaciones fijas; esta condición no podría adoptarse en un caso práctico ya que serían de esperarse importantes esfuerzos debidos a variaciones de temperatura, lo cual puede ser subsanado, como ya se mencionó con anterioridad, mediante el empleo de conectores sísmicos o amortiguadores viscosos.

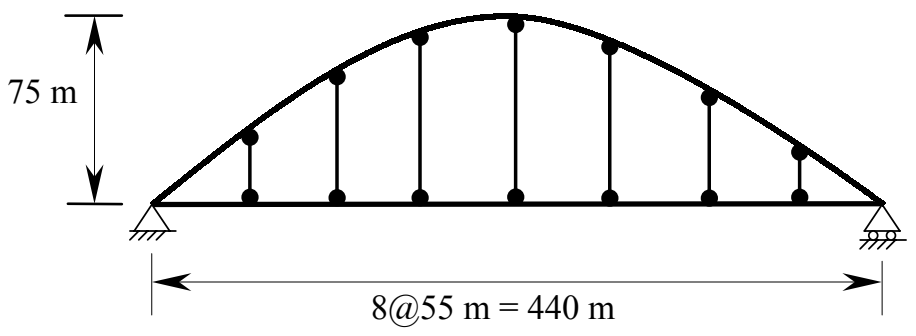

a) Puente arco con tablero inferior

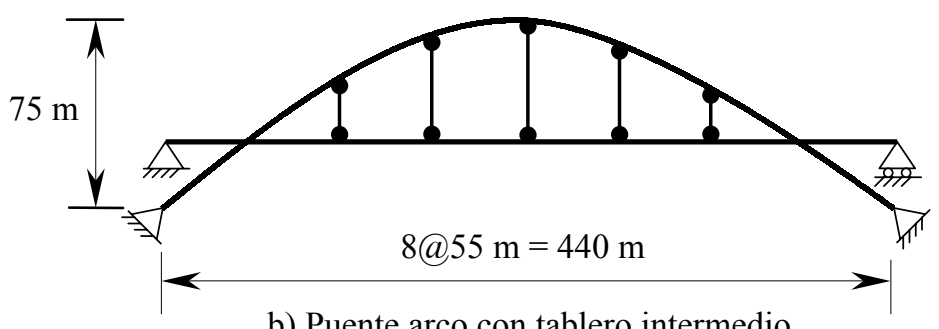

b) Puente arco con tablero intermedio

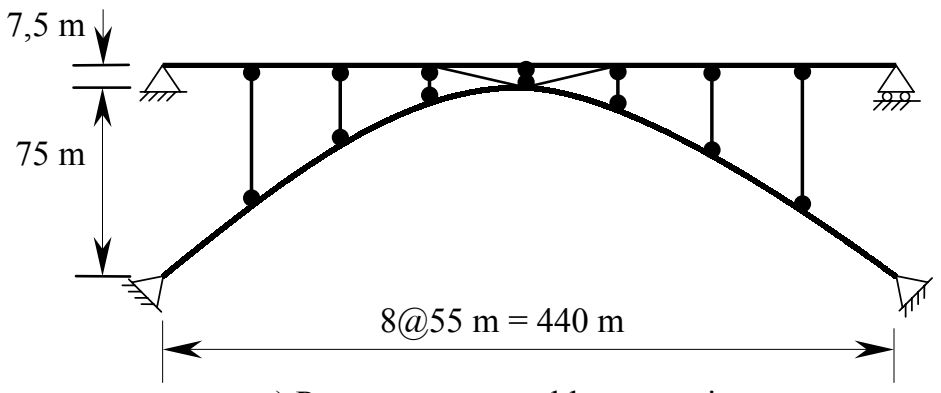

c) Puente arco con tablero superior

Figura 7. Modelos de tres puentes de gran claro (Nazmy, 1996) 


\section{Respuesta sísmica de tres puentes de gran claro}

Otro estudio similar al anterior fue realizado por Nazmy (1996), analizando en esta ocasión la respuesta dinámica de tres puentes arco: con tablero inferior, con tablero intermedio y con tablero superior, cada uno con un claro de $440 \mathrm{~m}$. En la fig. 7 se muestra la geometría en elevación de los modelos analíticos. Para los modelos con tablero inferior e intermedio, éste se consideró rígidamente conectado al arco. Todos los elementos verticales que conectaban el tablero y el arco se supusieron capaces de soportar únicamente fuerzas axiales de tensión o compresión. En el modelo con tablero superior se colocaron barras inclinadas que conectaban la clave del arco con el tablero, con la finalidad de transmitir la fuerza longitudinal entre ambos componentes. El ancho de los tres modelos se supuso igual a $20 \mathrm{~m}$.

Al igual que en la investigación anterior, en el análisis fueron consideradas la no linealidad debida al cambio de geometría producido por los desplazamientos, la interacción fuerza axial-momento flexionante, y la degradación de rigidez debida a la fuerza axial que actuaba en el arco, producto de la carga muerta.

Se compararon las respuestas ante excitaciones uniforme y múltiple de los apoyos, utilizando como señales de entrada dos acelerogramas del sismo de El Centro de 1979. Los desplazamientos nodales totales se descompusieron en desplazamientos cuasi-estáticos y relativos. Los cuasi-estáticos eran los originados por el movimiento no uniforme de los apoyos en cualquier instante. La fig. 8 muestra la comparación antes descrita para el desplazamiento vertical de la clave del arco para el puente con tablero superior; cada gráfica muestra la respuesta relativa y la total (relativa más cuasi-estática). Resultó evidente que la excitación múltiple en los apoyos tuvo un efecto significativo en el incremento de la respuesta al excitar más modos de vibrar debido al movimiento fuera de fase de los apoyos.

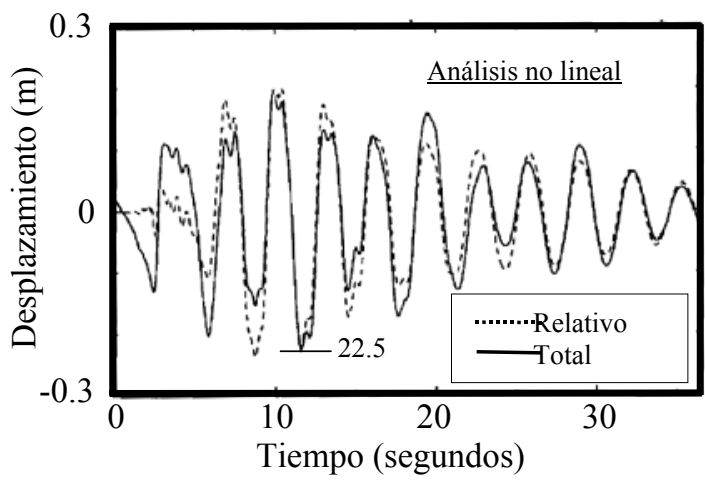

a) Respuesta ante excitación uniforme

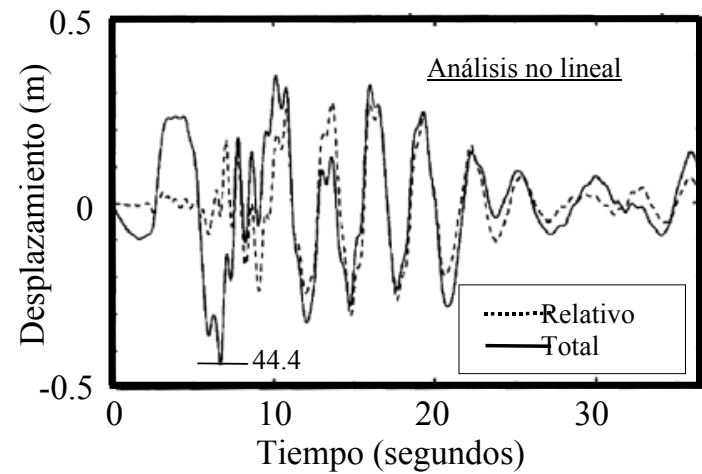

b) Respuesta ante excitación múltiple

Figura 8. Historia de desplazamientos verticales de la clave del arco del puente con tablero superior (escaneada de Nazmy, 1996)

Vale la pena señalar que los extremos del tablero de los modelos de la fig. 7 se supusieron apoyados en una articulación fija y en un rodillo. Esto tiene el inconveniente de que gran parte de la acción sísmica se concentra en la zona del tablero adyacente a la articulación (fig. 2.b). 


\section{PUENTES DE CONCRETO}

\section{El Puente Nagayabu}

Cronológicamente los puentes de concreto han sido estudiados, en lo que a comportamiento sísmico se refiere, posteriormente a los de acero. Uno de los primeros trabajos que aparecen en la literatura consultada es el debido a Horinouchi et al. (1996), quienes estudiaron el puente Nagayabu, de concreto reforzado, construido in situ sobre cimbra. En la fig. 9 se muestra la geometría del puente. Cuenta con una longitud total de $140 \mathrm{~m}$, y un arco con claro de $82.8 \mathrm{~m}$ y $23.9 \mathrm{~m}$ de flecha, para una relación $\mathrm{f} / \mathrm{L}=1 / 3.46$. La estructura está formada por una losa aligerada de $7.6 \mathrm{~m}$ de anchura total y $1 \mathrm{~m}$ de espesor, un arco laminar de $5.5 \mathrm{~m}$ de anchura por $1.2 \mathrm{~m}$ de espesor y pilas formadas por dos elementos de sección rectangular de $1.75 \mathrm{~m}$ por $0.8 \mathrm{~m}$, cada una.

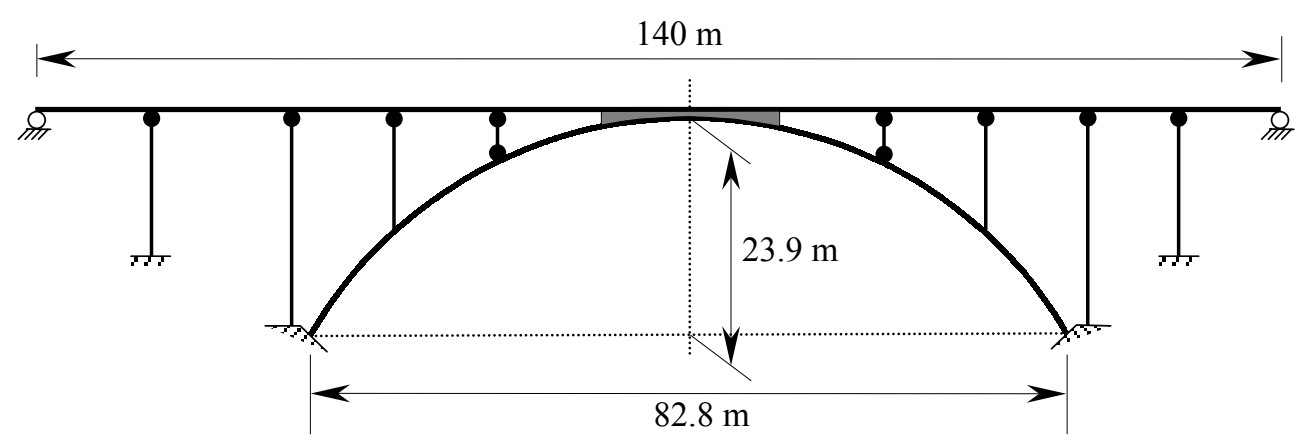

a) Vista en elevación

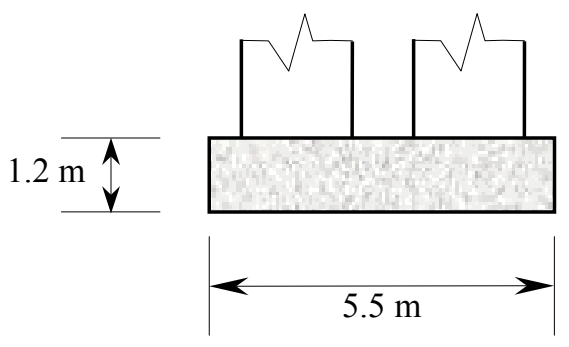

b) Sección transversal del arco

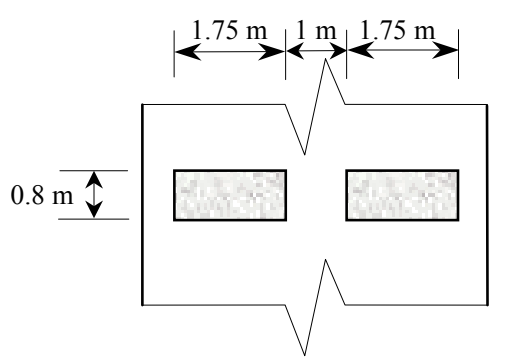

c) Sección de pilas sobre el arco

Figura 9. Geometría del puente Nagayabu (Horinouchi et al., 1996)

El puente fue diseñado de acuerdo con la normas japonesas de la época, mediante un análisis modal espectral. De acuerdo con dichas normas la aceleración espectral de diseño estaba dada por

$$
S=C_{Z} \cdot C_{I} \cdot C_{D} \cdot S_{O}
$$

en donde,

$\mathrm{S}=$ aceleración espectral de diseño,

$\mathrm{C}_{\mathrm{Z}}=$ factor de ubicación regional $=0.85$ (zona $\left.\mathrm{B}\right)$,

$\mathrm{C}_{\mathrm{I}}=$ factor de importancia del camino $=1$ (primer grado), 
$\mathrm{C}_{\mathrm{D}}=$ factor de amortiguamiento $=1$, para un coeficiente de amortiguamiento del $5 \%, \mathrm{y}$ $\mathrm{S}_{\mathrm{O}}=$ aceleración espectral básica para terreno tipo 1 .

La aceleración espectral básica se obtuvo para dos casos:

- Caso 1: la aceleración del espectro de respuesta especificado en la norma japonesa de la época, y

- Caso 2: la aceleración del espectro de respuesta del sismo de Hyogo-ken Nanbu en 1995, obtenido en el Observatorio Meteorológico de Kobe.

Con fines comparativos, se realizó un análisis modal espectral para ambos casos, considerando un comportamiento lineal de la estructura. En la tabla 5 se presentan los periodos y las aceleraciones espectrales para los 10 primeros modos. Como se observa, algunos periodos están muy cercanos entre sí (modos 8 y 9), y para el caso 2 se obtuvieron pseudoaceleraciones hasta diez veces mayores que las del caso 1 en dirección horizontal (modos 2, 3 y 4), y hasta 13 veces mayores en dirección vertical (modo 6). Como consecuencia de lo anterior, se obtuvo que los elementos mecánicos en los arranques del arco y en el tablero para el caso 2 resultaron aproximadamente diez veces mayores que los del caso 1. Cabe hacer notar que el epicentro del sismo de Hyogo-ken Nanbu se localizó a tan sólo $20 \mathrm{~km}$ del Observatorio Meteorológico de Kobe, y que ante excitaciones tan intensas como éstas es necesario realizar un análisis no lineal para poder verificar la integridad estructural del puente.

\begin{tabular}{|c|c|c|c|c|c|c|}
\hline \multirow{3}{*}{ Modo } & \multirow{3}{*}{$\begin{array}{l}\text { Periodo } \\
\text { (s) }\end{array}$} & \multirow{3}{*}{$\begin{array}{l}\text { Dirección } \\
\text { principal del } \\
\text { movimiento }\end{array}$} & \multicolumn{4}{|c|}{ Aceleración espectral (gals) } \\
\hline & & & \multicolumn{2}{|c|}{ Caso 1} & \multicolumn{2}{|c|}{ Caso 2} \\
\hline & & & Horizontal & Vertical & Horizontal & Vertical \\
\hline 1 & 1.072 & En el plano & 170 & 85 & 1,275 & 425 \\
\hline 2 & 0.695 & Fuera del plano & 170 & 85 & 1,700 & 510 \\
\hline 3 & 0.446 & En el plano & 170 & 85 & 1,700 & 595 \\
\hline 4 & 0.330 & Fuera del plano & 170 & 85 & 1,700 & 595 \\
\hline 5 & 0.281 & En el plano & 170 & 85 & 1,530 & 850 \\
\hline 6 & 0.252 & Fuera del plano & 170 & 85 & 1,275 & 1,105 \\
\hline 7 & 0.221 & En el plano & 170 & 85 & 1,020 & 935 \\
\hline 8 & 0.194 & En el plano & 170 & 85 & 850 & 850 \\
\hline 9 & 0.188 & En el plano & 170 & 85 & 850 & 850 \\
\hline 10 & 0.159 & Fuera del plano & 170 & 85 & 850 & 850 \\
\hline
\end{tabular}

\section{El Puente Bixby Creek}

En la década de los 30's del siglo pasado se construyeron varios puentes arco de concreto reforzado a lo largo de la costa de California, mismos que han sido considerados como verdaderos ejemplos de eficiencia, economía y elegancia (Billington, 1977). Sin embargo, estas estructuras han venido a quedar sísmicamente deficientes ante los códigos actuales de diseño. Recientemente, el California Department of Transportation (Caltrans) ha desarrollado un 
programa intensivo de evaluación sísmica y refuerzo de estos puentes arco. El puente Bixby Creek (fig. 10) formó parte de este programa; su longitud total es de aproximadamente $210.45 \mathrm{~m}$, con un claro del arco igual a $100.65 \mathrm{~m}$ y una flecha de $36.6 \mathrm{~m}$ (fig. 11). Mención especial merecen las numerosas juntas de dilatación con las que contaba originalmente el puente: todas las pilas se construyeron con su extremo superior dividido en dos partes, como se aprecia en el detalle de la fig. 11, por lo que el tablero era discontinuo. En reconocimiento a la vulnerabilidad sísmica de las pilas así construidas, previamente a este estudio Caltrans reforzó las juntas con un encamisado formado por placas de acero ancladas a los cabezales de la pilas, para intentar restringir los desplazamientos excesivos que pudieran presentarse a través de las juntas.
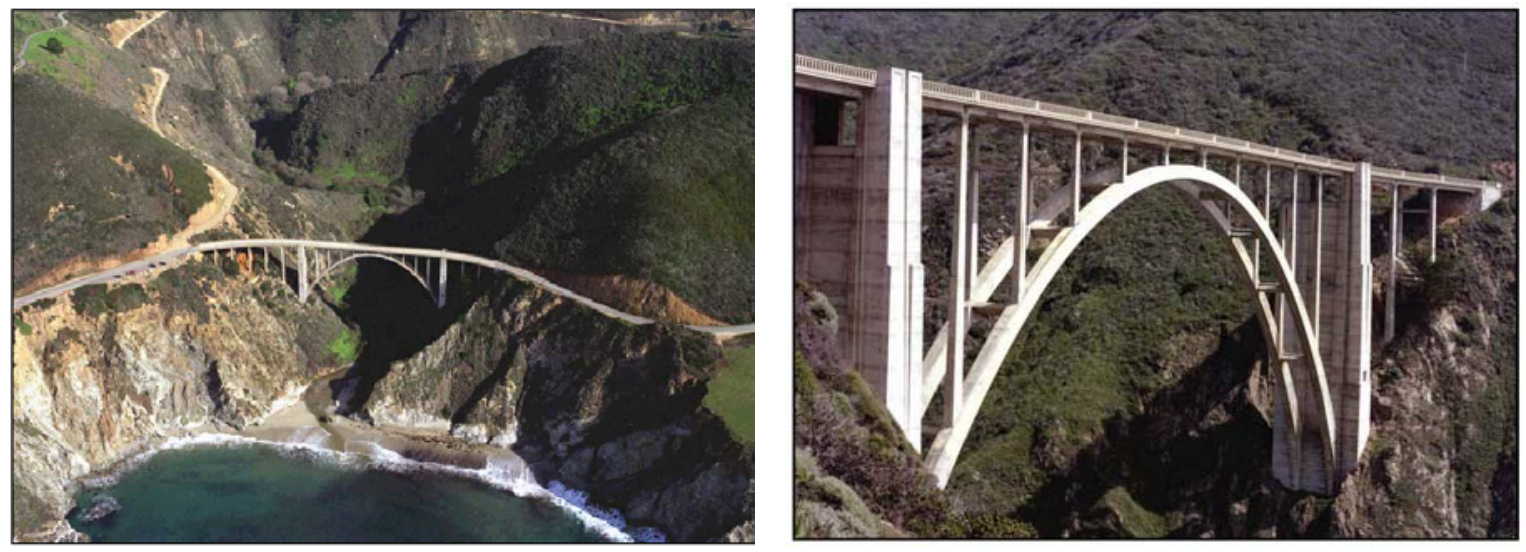

Figura 10. El Puente Bixby Creek (McCallen et al., 1999)

Uno de los objetivos principales del estudio era investigar la respuesta no lineal del puente ante excitación sísmica severa, por lo que se elaboró un detallado modelo tridimensional no lineal de elementos finitos que permitiese considerar tanto la no linealidad geométrica como la de los materiales (McCallen et al., 1999). La no linealidad del concreto y del acero de refuerzo se consideró para los arcos y las pilas; el resto de los elementos se consideraron elásticos. Mediante elementos discretos se modeló el posible contacto e impacto a través de las juntas de dilatación del tablero, lo que provocaba una no linealidad geométrica cuando la estructura era sometida a movimientos fuertes, ya que dichas juntas podían abrirse o cerrarse, o inclusive ocurrir impacto. El encamisado metálico también fue tomado en cuenta.

Las propiedades dinámicas fundamentales de la estructura se determinaron mediante mediciones de vibración en el sitio y analíticamente. Las observaciones de campo identificaron claramente el modo fundamental transversal con un periodo de 2.5 segundos, valor que permitió validar el modelo analítico. Las simulaciones analíticas incluyeron varias idealizaciones de las conectividades en las juntas de dilatación que se localizaban en el cabezal de cada pila, así como un análisis estático no lineal para las cargas muertas previo al cálculo de las propiedades dinámicas. Los periodos fundamentales calculados variaron entre 1.17 y 3.86 segundos, dependiendo de la idealización de las juntas de dilatación del modelo. Para refinar el modelo se agregó una idealización más, que consistió en suponer que en el primer modo de vibrar en el que el tablero se desplazaba transversalmente, las juntas de un lado de éste se abrían mientras que las 
del otro entraban en contacto. Para este caso, el periodo calculado del modo fundamental fue de 2.6 segundos, como se ilustra en la fig. 12.

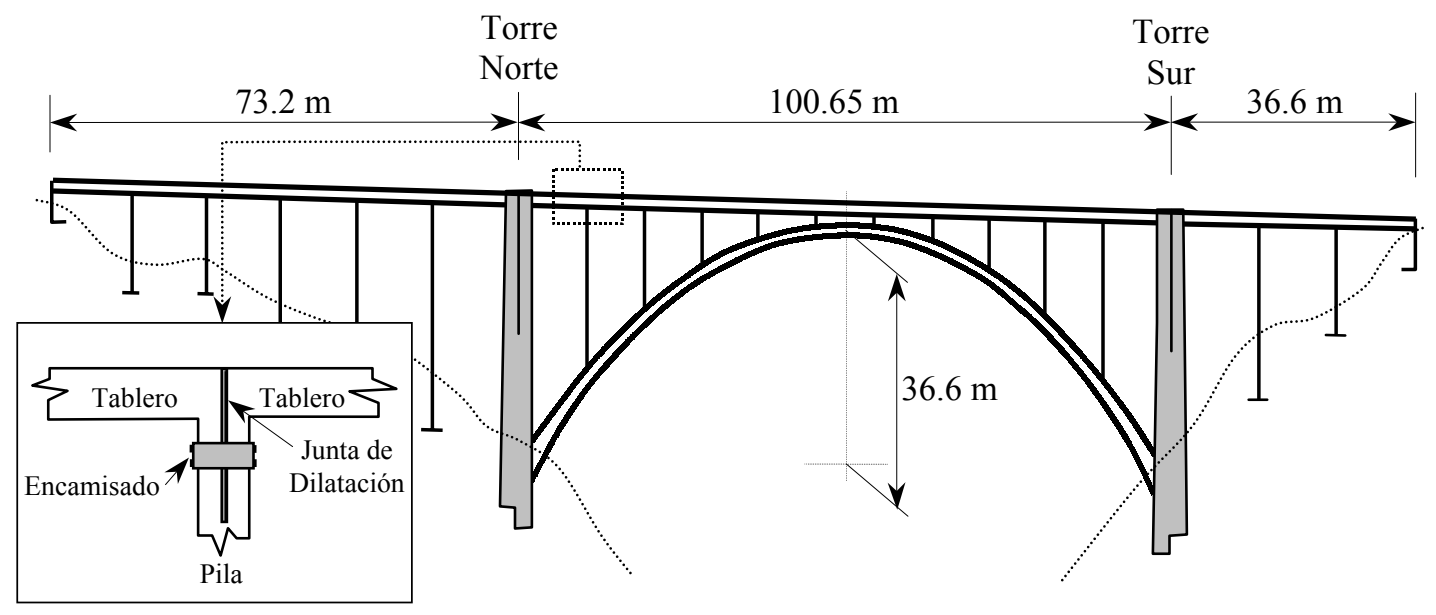

Figura 11. Geometría del Puente Bixby Creek, vista en elevación

Para evaluar la influencia del movimiento diferencial de los apoyos del puente se estudiaron los efectos topográficos del sitio mediante un modelo bidimensional de elementos finitos. Los resultados indicaron que la topografía del cañón comenzaba a tener influencia en el movimiento del suelo para frecuencias del orden de 2 a $3 \mathrm{~Hz}$; para 5 a $7 \mathrm{~Hz}$ los efectos de refracción de las ondas ya eran bastante pronunciados. Mediciones de sismos pequeños confirmaron estas observaciones; el movimiento diferencial entre las torres norte y sur era apreciable para frecuencias de $2 \mathrm{~Hz}$ en adelante y para $5 \mathrm{~Hz}$ las torres estaban claramente fuera de fase. Dado que el puente era bastante flexible (con un periodo fundamental de 2.5 segundos), se decidió que el movimiento variable de los apoyos no constituía, en este caso, un factor a considerar en el comportamiento de la estructura, ya que sólo afectaría a los modos superiores.

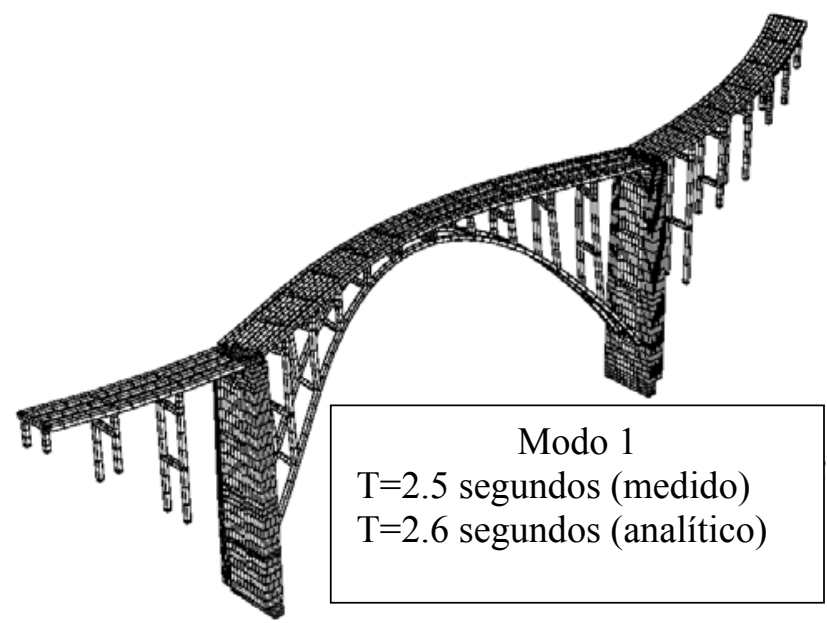

Figura 12. Modo fundamental transversal del Puente Bixby Creek (McCallen et al., 1999) 
La falla de Palo Colorado-San Gregorio pasa aproximadamente a $1 \mathrm{~km}$ del sitio donde se localiza el puente, y a ella se le atribuye un magnitud máxima esperada de 7.5, una aceleración horizontal pico de $0.72 \mathrm{~g}$ y de $0.70 \mathrm{~g}$ para la aceleración vertical. Para evaluar la respuesta sísmica del puente se generaron tres grupos de acelerogramas sintéticos. El tercero de ellos, que resultó el más desfavorable en la respuesta sísmica del puente, incluyó las componentes de movimiento del terreno de fuente cercana, que pueden dar lugar a pulsos de desplazamiento de periodo largo y que tienen una considerable influencia en la degradación estructural (Kawashima, 2000; Lobo, 2000); se generaron tres señales diferentes, que se aplicaron simultáneamente a los diferentes apoyos del modelo del puente: normal a la falla, paralela a la falla y vertical. En la fig. 13 se muestran el acelerograma y el desplacigrama para la componente normal a la falla.
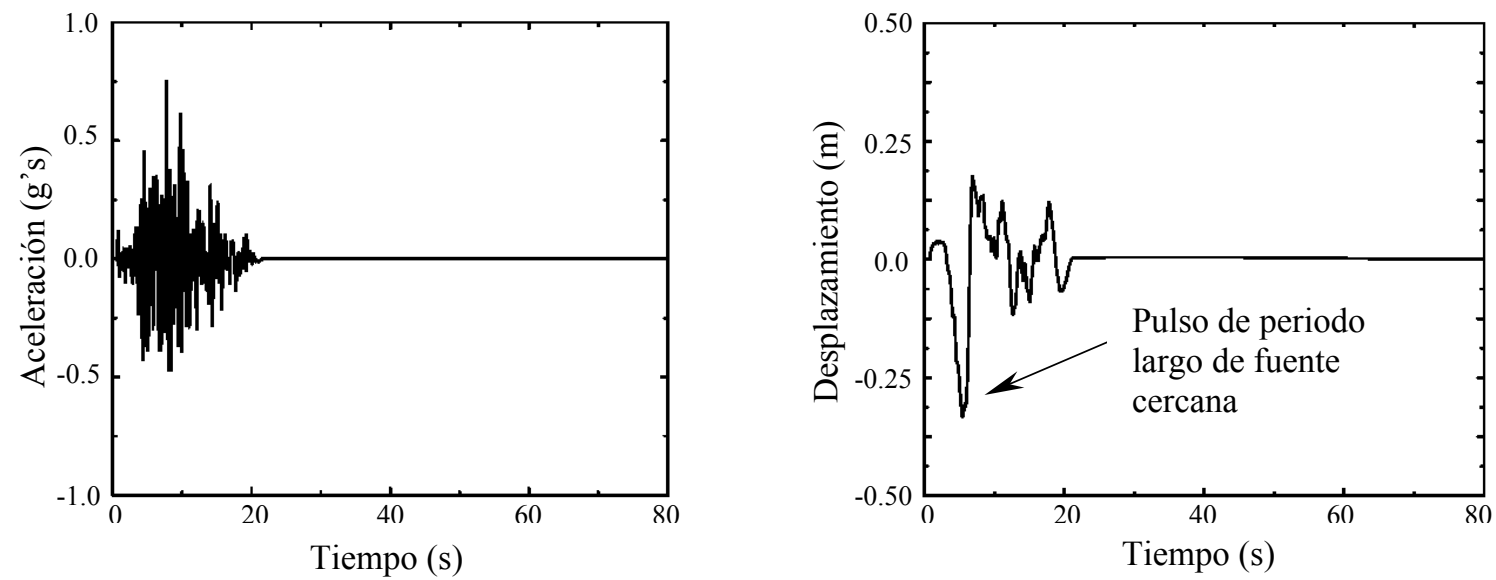

Figura 13. Señales sintéticas de la componente normal a la falla para un sismo de magnitud 7.5, incluyendo efectos de fuente cercana (McCallen et al., 1999)

La respuesta desplazamiento transversal-tiempo para la clave del arco del modelo no lineal de la estructura ante la excitación sísmica descrita se muestra en la fig. 14. Se aprecia que el puente respondió violentamente al pulso de desplazamiento al inicio del movimiento (aproximadamente a los 7 segundos), con un desplazamiento máximo de casi $0.90 \mathrm{~m}$, lo cual es excesivo para un puente de $100 \mathrm{~m}$ de claro principal. Como se aprecia en la fig. 13, el movimiento del suelo cesó aproximadamente a los 20 segundos, por lo que de ahí en adelante, en la fig. 14 la estructura oscilaba en movimiento libre; se aprecia que el periodo se incrementó a más de 6 segundos debido a la degradación sufrida por la estructura. En algunos elementos se desarrollaron altas deformaciones en el concreto, excediendo las permitidas por Caltrans (0.005); las deformaciones del acero de refuerzo no excedieron en ningún sitio a la permisible por Caltrans (0.12), aunque la fluencia del mismo se presentó en diversos elementos.

Anteriormente a la realización de este estudio se llevó a cabo un proyecto de refuerzo del puente Bixby Creek, del cual destaca el presfuerzo longitudinal del tablero rellenando las juntas de dilatación con grout, con la idea de que el tablero funcionase como una viga de gran peralte ante cargas transversales. Para la estructura reforzada también se elaboraron modelos no lineales que permitiesen evaluar su comportamiento. Los análisis mostraron un sustancial incremento en 
la rigidez del sistema, lo cual condujo a un dramático decremento de los desplazamientos calculados de la estructura.

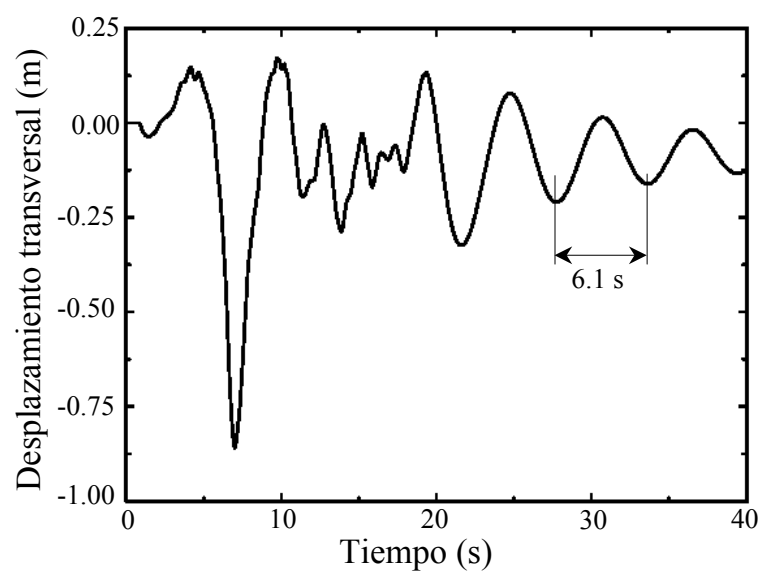

Figura 14. Respuesta de la clave del arco considerando no linealidad geométrica y de los materiales, e incluyendo contacto en las juntas de dilatación (McCallen et al., 1999)

Los puentes arco de gran claro tienden a ser sistemas muy flexibles, con periodos fundamentales relativamente largos (las mediciones de campo mostraron un periodo fundamental de 2.5 segundos para el puente en estudio). Lo anterior sugiere la necesidad de incluir pulsos de desplazamiento de fuente cercana en la evaluación del peligro sísmico si la estructura se localiza cerca de una falla activa.

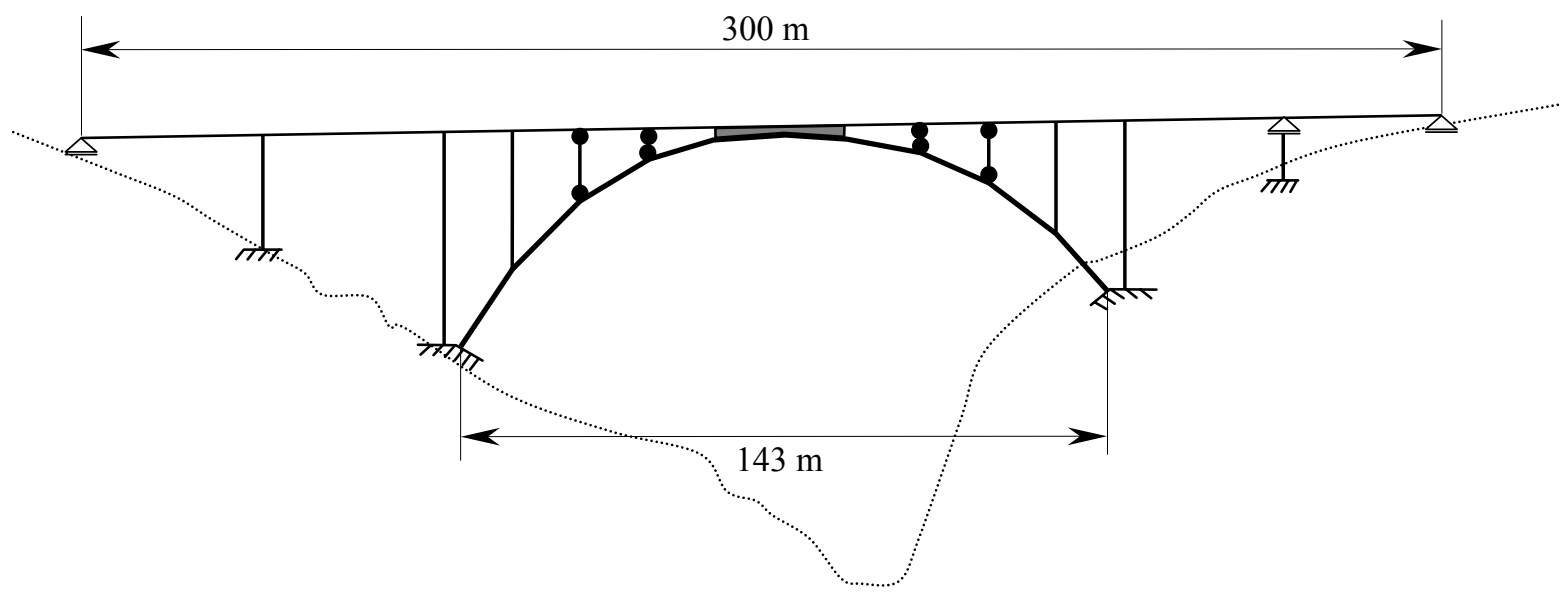

Figura 15. Geometría del puente sobre la cañada Takachio (Nakagawa et al., 2000)

\section{Puente sobre la cañada Takachio}

El puente diseñado por Nakagawa et al. (2000) sería construido sobre la cañada Takachio, dentro de un parque nacional de Japón; contaría con una longitud total de $300 \mathrm{~m}$ y un claro principal de $143 \mathrm{~m}$. Se trata de un puente arco de concreto reforzado con un tablero de sección en cajón de 
concreto presforzado de $15.5 \mathrm{~m}$ de anchura total. En la fig. 15 se muestra la geometría en elevación del modelo.

\begin{tabular}{|c|c|c|c|}
\hline \multicolumn{4}{|c|}{$\begin{array}{c}\text { Tabla 6. Resultados del análisis modal en dirección } \\
\text { longitudinal (Nakagawa et al., 2000) }\end{array}$} \\
\hline Modo & $\begin{array}{c}\text { Periodo natural } \\
\text { (segundos) }\end{array}$ & $\begin{array}{c}\text { Factor de } \\
\text { participación modal }\end{array}$ & $\begin{array}{c}\text { Masa efectiva } \\
\text { acumulada (\%) }\end{array}$ \\
\hline 1 & 1.473 & 36.960 & 61 \\
\hline 2 & 0.701 & 1.031 & 61 \\
\hline 3 & 0.451 & 5.129 & 62 \\
\hline 4 & 0.376 & -2.053 & 62 \\
\hline 5 & 0.314 & 3.455 & 63 \\
\hline 6 & 0.263 & -1.470 & 68 \\
\hline 7 & 0.257 & 7.296 & 71 \\
\hline 8 & 0.249 & 13.680 & 79 \\
\hline
\end{tabular}

De acuerdo con las normas (Japan Road Association, 1996), el puente se clasificó dentro del grupo B (puentes de importancia alta), sería desplantado sobre suelo tipo I (roca), y se ubicaría regionalmente en la zona B (riesgo moderado). Para el arco y las pilas con conexiones rígidas en ambos extremos se consideró el comportamiento no lineal del concreto y del acero de refuerzo. Para estas barras se supuso un factor de amortiguamiento del $2 \%$, mientras que para las barras con comportamiento lineal se supuso del $5 \%$.

Para fines de diseño se llevó a cabo un análisis dinámico no lineal en dirección longitudinal, modelando el puente como un marco plano. Como excitaciones sísmicas se consideraron tres diferentes aceleraciones de periodo largo (sismo Tipo I, producto de movimientos en el borde de la placa tectónica) y tres de periodo corto (Tipo II, producto de sismos intraplaca), con amplitudes de $3.30 \mathrm{~m} / \mathrm{s}^{2}$ y $6.30 \mathrm{~m} / \mathrm{s}^{2}$, y una duración de 20 segundos.

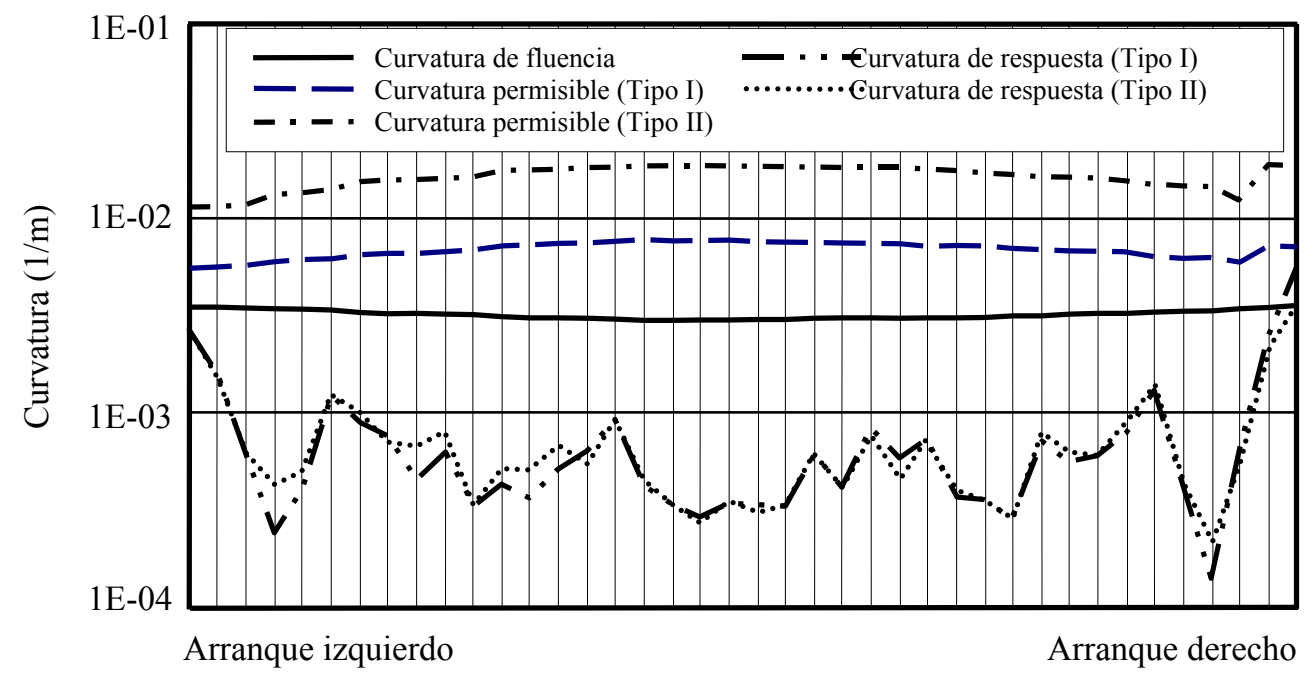

Figura 16. Curvaturas permisibles y calculadas para el arco (Nakagawa et al., 2000) 
Las propiedades dinámicas de la estructura en dirección longitudinal se presentan en la tabla 6 para los primeros ocho modos, habiéndose considerado el efecto de la fuerza axial debida a carga muerta en pilas y arco en el cálculo de las mismas. Como se aprecia, para este caso la respuesta estuvo dominada por el modo fundamental.

Se llevó a cabo un análisis dinámico no lineal de la respuesta usando relaciones momento flexionante-curvatura de los elementos estructurales considerando la fuerza axial debida a carga muerta. Los análisis indicaron que las curvaturas resultantes en los diferentes elementos estaban por debajo de los valores permisibles. En la fig. 16 se muestran las curvaturas permisibles y las obtenidas en la respuesta para el arco, despreciando el efecto de la fluctuación de la fuerza axial durante el movimiento. Únicamente en el arranque derecho del arco se sobrepasó la curvatura de fluencia.

Cuando se toma en cuenta la fluctuación de la carga axial durante el movimiento sísmico, es de esperarse que ocurran cambios en las relaciones momento flexionante-curvatura. Para estudiar este efecto se calcularon las curvaturas permisibles y las de respuesta, para tres condiciones de carga: fuerza axial debida a carga muerta, fuerza axial máxima debida a carga muerta más sismo y fuerza axial mínima. Un resumen de los resultados para el arranque derecho del arco (el más desfavorable) sometido a la excitación Tipo I (de periodo largo) se muestra en la fig. 17. Como se aprecia, las curvaturas de respuesta resultaron menores que las permisibles.
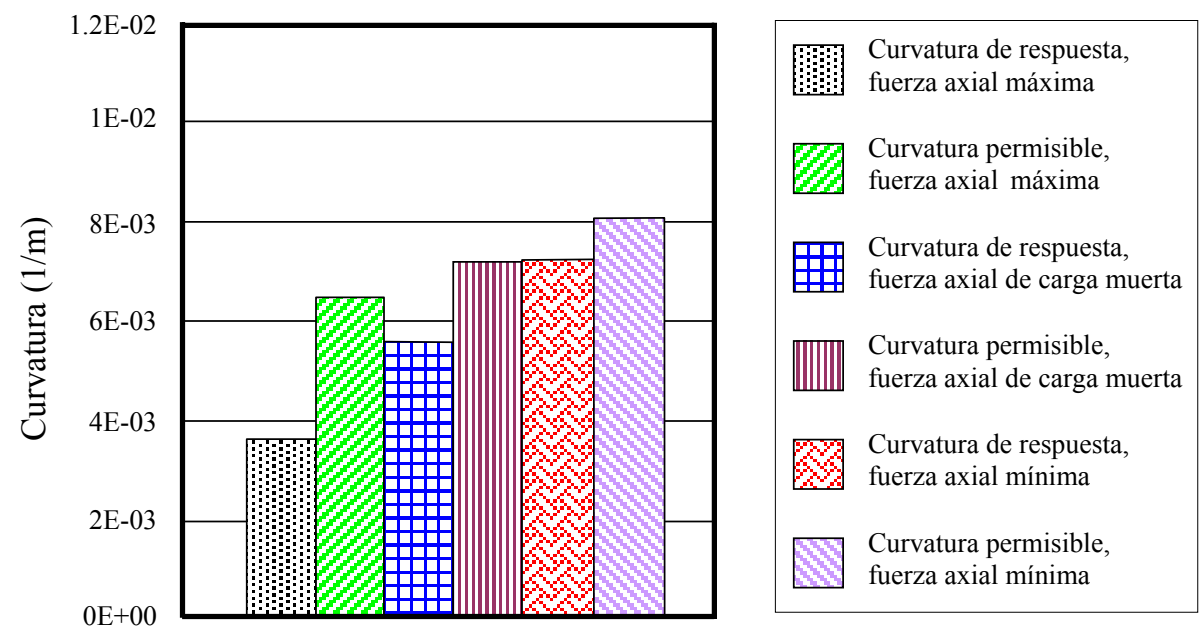

Figura 17. Curvaturas en el arranque derecho del arco para la excitación sísmica Tipo I

(Nakagawa et al., 2000)

\section{Evaluación de un puente existente sometido al sismo de Hyogo-ken Nanbu}

Kawashima y Mizoguti (2000) estudiaron la respuesta sísmica de un puente de concreto reforzado sometido a la fuerte excitación del sismo de Hyogo-ken Nanbu de 1995, estructura que fue proyectada de acuerdo al método estático basado en el diseño por esfuerzos permisibles según las normas japonesas, con un coeficiente sísmico de 0.18 tanto en dirección longitudinal como 
transversal. La geometría del modelo se presenta en la fig. 18. Se trata de un puente carretero de dos carriles, con un tablero de $9.5 \mathrm{~m}$ de anchura, un claro principal de $150 \mathrm{~m}$ y una flecha del arco de $27 \mathrm{~m}$, para una relación flecha/claro de 1/5.6. La sección transversal del arco es un cajón bicelular de concreto reforzado.

La cimentación del puente está desplantada sobre granito intemperizado, con velocidades de propagación de onda de aproximadamente $700 \mathrm{~m} / \mathrm{s}$ del lado izquierdo y $1400 \mathrm{~m} / \mathrm{s}$ del lado derecho del puente. El terreno se clasificó como Tipo I, de acuerdo con las especifícaciones japonesas (Japan Road Association, 1996).

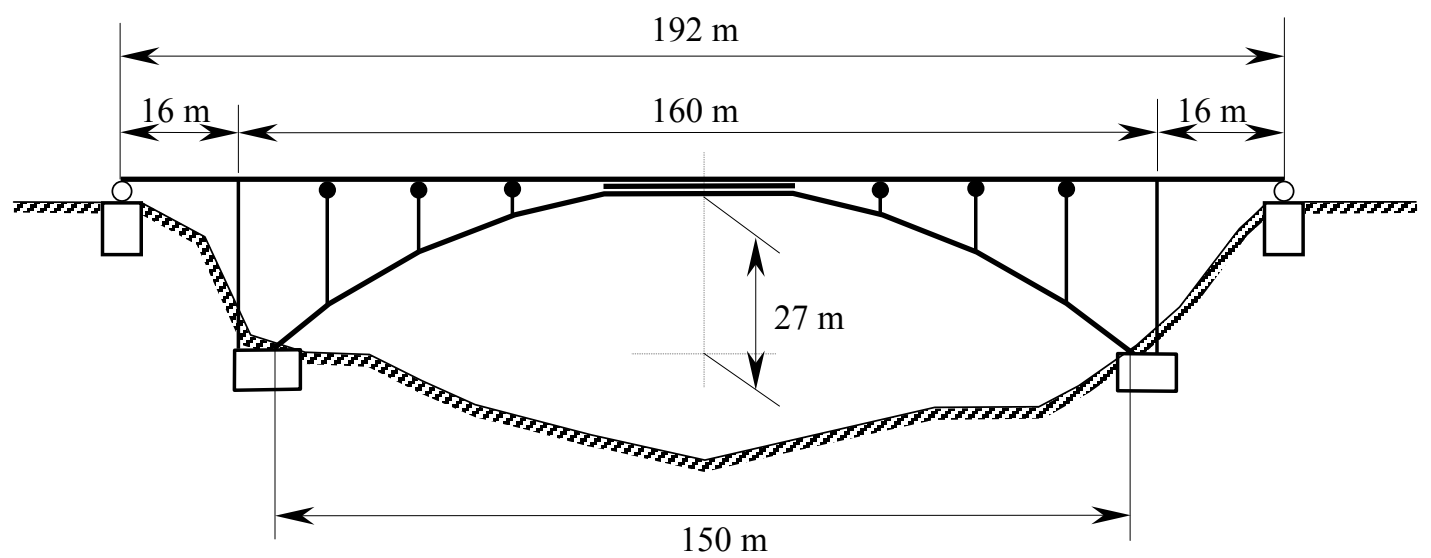

Figura 18. Geometría del modelo. Vista en elevación (Kawashima y Mizoguti, 2000)

El puente se modeló mediante elementos barra, como se aprecia en la fig. 18. El tablero y las pilas se idealizaron como barras con comportamiento lineal. Resultaría importante considerar la interacción no lineal de la fuerza axial y el momento flexionante en el arco, dado que sería de esperarse que ocurriesen grandes variaciones de la fuerza axial; sin embargo, como no se contaba con un modelo analítico confiable que tomase en cuenta esta importante variación de la fuerza axial, solamente se consideró la no linealidad en el comportamiento a flexión, por medio de un modelo histerético elastoplástico.

\begin{tabular}{|c|c|c|c|}
\hline \multicolumn{5}{|c|}{$\begin{array}{c}\text { Tabla 7. Propiedades dinámicas del modelo } \\
\text { (Kawashima y Mizoguti, 2000) }\end{array}$} \\
\hline Modo & $\begin{array}{c}\text { Periodo natural } \\
\text { (segundos) }\end{array}$ & $\begin{array}{c}\text { Factor de } \\
\text { participación modal }\end{array}$ & $\begin{array}{c}\text { Masa efectiva } \\
\text { acumulada (\%) }\end{array}$ \\
\hline 1 & 2.000 & 13.63 & 26 \\
\hline 2 & 1.070 & 0 & 26 \\
\hline 3 & 0.612 & -4.68 & 29 \\
\hline 4 & 0.447 & 0 & 29 \\
\hline 5 & 0.338 & -10.7 & 45 \\
\hline 6 & 0.317 & 0 & 45 \\
\hline 7 & 0.234 & 0 & 45 \\
\hline 8 & 0.229 & 2.58 & 46 \\
\hline
\end{tabular}


En la tabla 7 se presentan las propiedades dinámicas para los primeros ocho modos de vibrar en el plano del puente. En la última columna de la tabla se observa que la masa efectiva acumulada del primero al octavo modos sólo fue del $46 \%$ de la masa total, lo cual indica la importancia de incluir los modos superiores en la evaluación de la respuesta.
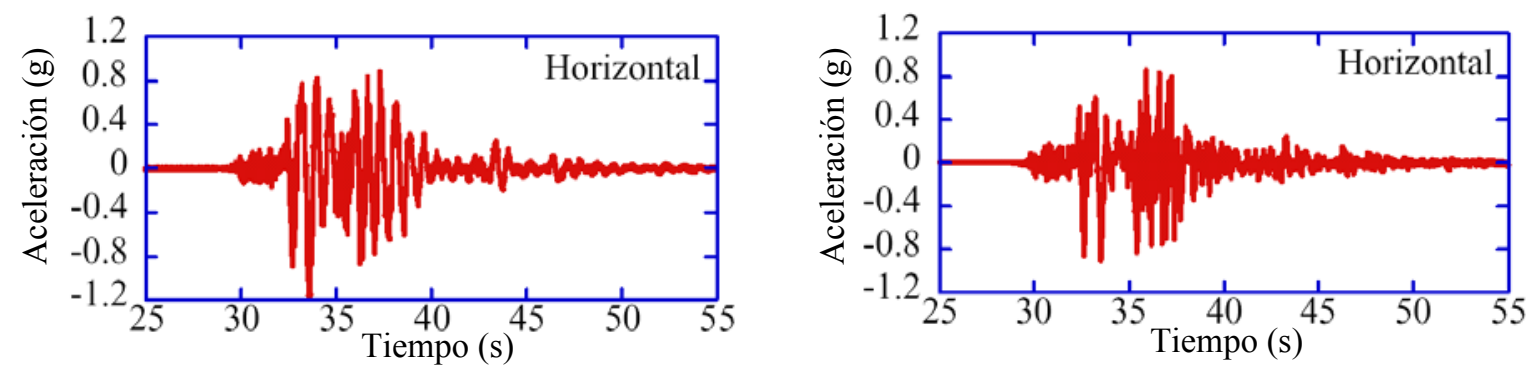

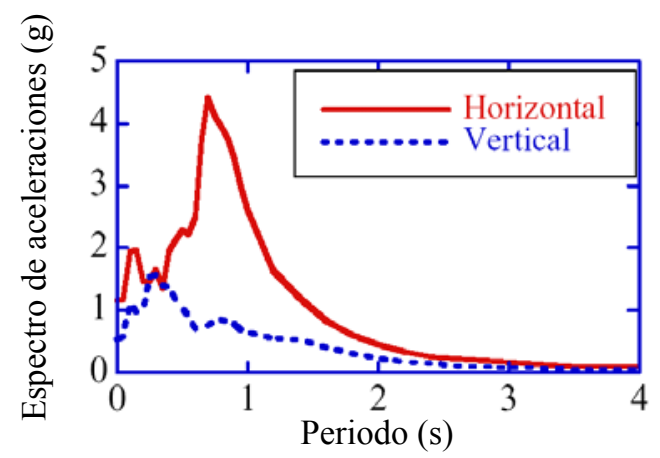

a) Lado izquierdo de la cimentación

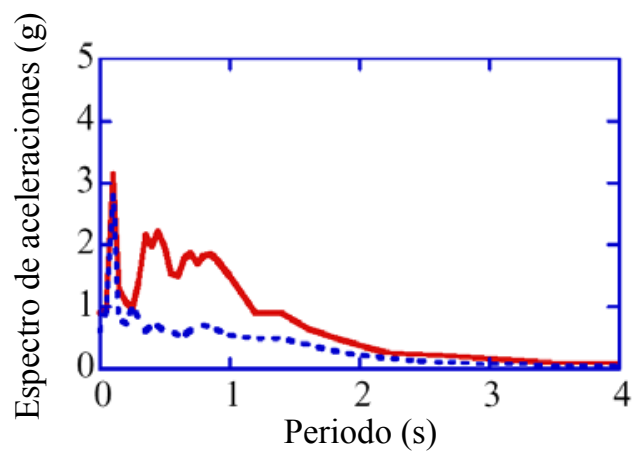

b) Lado derecho de la cimentación

Figura 19. Aceleraciones del terreno calculadas para ambos extremos del puente (Kawashima y Mizoguti, 2000)

Los acelerogramas de las componentes N-S y vertical registrados por el Observatorio de Kobe se usaron como señales de entrada. Mediante un proceso de deconvolución de las dos señales registradas se obtuvieron las aceleraciones en la base rocosa; posteriormente se aplicaron estas historias de aceleración a un modelo bidimensional de elementos finitos del terreno circundante al puente para calcular las aceleraciones del terreno de las cimentaciones izquierda y derecha. En la fig. 19 se muestran los acelerogramas calculados para ambos apoyos del puente en dirección horizontal, así como los espectros de respuesta de aceleraciones en direcciones horizontal y vertical para un factor de amortiguamiento de 0.05 .

Para comprender las características básicas de la respuesta del puente, primeramente se analizó el comportamiento ante excitación horizontal uniforme, empleando los acelerogramas calculados para el lado derecho (fig. 19.b). La máxima aceleración lateral de $0.8 \mathrm{~g}$ ocurrió en ambos arranques, mientras que el máximo desplazamiento lateral de $0.15 \mathrm{~m}$ ocurrió a $1 / 4$ y a $3 / 4$ del claro del arco. Cabe hacer notar que una aceleración de $1 \mathrm{~g}$ y un desplazamiento de aproximadamente $0.3 \mathrm{~m}$ ocurrió en dirección vertical a pesar de que la excitación se dio sólo en 
dirección horizontal. Esto se debe a un acoplamiento modal significativo de la estructura en las direcciones horizontal y vertical.

En seguida, además de la excitación horizontal se agregó la excitación vertical del terreno de manera uniforme a ambos apoyos del puente. La respuesta vertical se vio incrementada de manera importante al agregar la excitación vertical; por ejemplo, en el arranque izquierdo del arco aumentó la compresión máxima de $60 \mathrm{MN}$ a $70 \mathrm{MN}$ y la mínima disminuyó de $20 \mathrm{MN}$ a 10 MN. El momento flexionante inducido por estas aceleraciones excedió el momento de diseño en diferentes secciones.

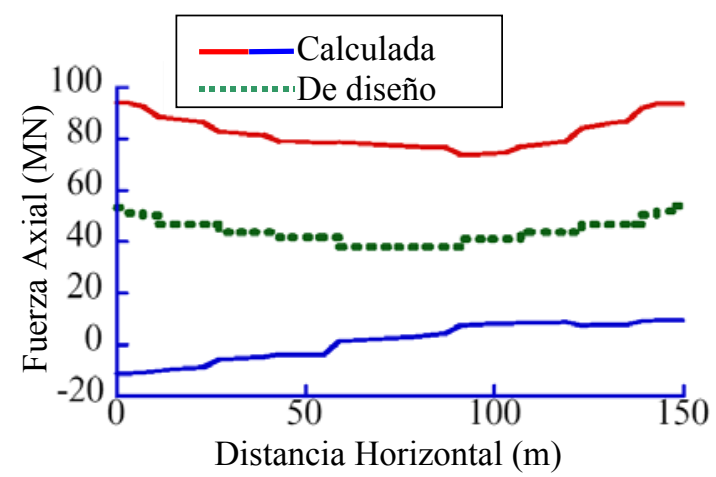

a) Fuerza axial

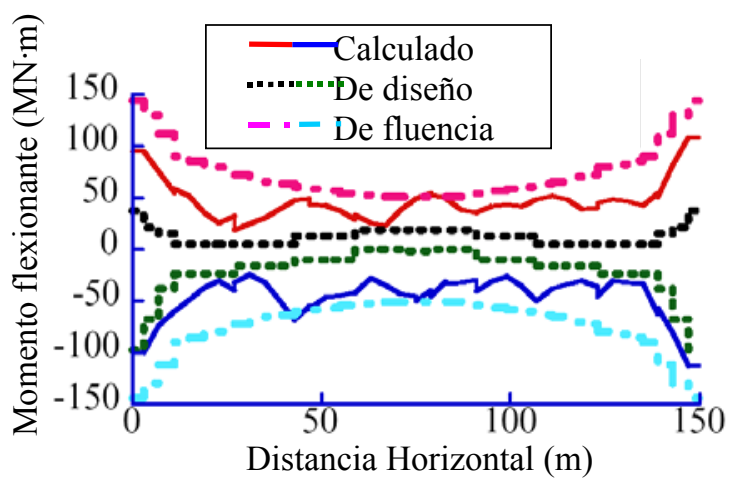

b) Momento flexionante

Figura 20. Fuerza axial y momento flexionante en el arco ante excitaciones múltiples de sus arranques (Kawashima y Mizoguti, 2000)

La respuesta más desfavorable del arco se presentó ante excitación múltiple, cuando se emplearon como señales de entrada para los arranques izquierdo y derecho los acelerogramas horizontal (fig. 19) y vertical. El arco experimentó aceleraciones horizontales máximas del orden de $1 \mathrm{~g}$, y aceleraciones verticales en riñones mayores que $2 \mathrm{~g}$; los mayores desplazamientos absolutos se presentaron en dirección vertical, alcanzando los $0.4 \mathrm{~m}$ también en riñones. En la fig. 20 se presentan las envolventes de la fuerza axial y del momento flexionante en el arco. La fuerza axial máxima prácticamente duplicó la de diseño, inclusive se presentó fuerza normal de tensión; además, llegó a desarrollarse fluencia en la clave del arco. Es importante hacer notar que estos resultados se presentaron en un arco diseñado de acuerdo al método estático, lo que sugiere la necesidad del empleo de técnicas más refinadas en el diseño de esta compleja tipología estructural.

\section{Puente de gran claro empleando amortiguadores viscosos}

Como ya se comentó en el resumen, los autores están llevando a cabo una investigación en la que se evalúa la respuesta sísmica de puentes equipados con amortiguadores viscosos. A continuación se presentan los primeros resultados de este estudio (Alvarez et al., 2002). 


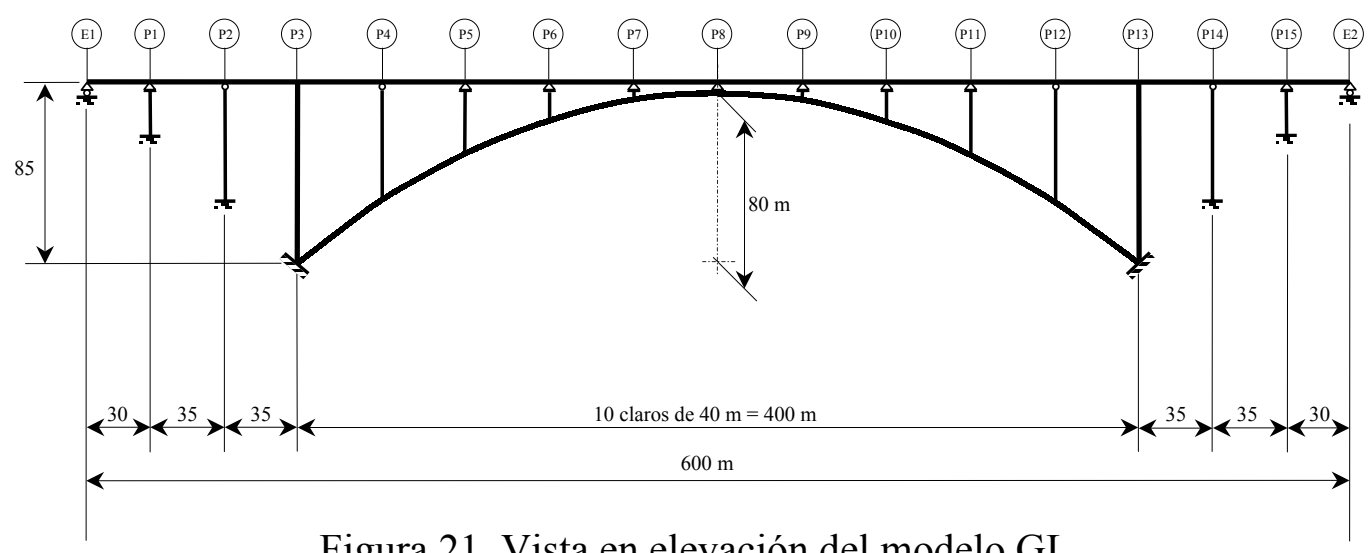

Figura 21. Vista en elevación del modelo GL

La fig. 21 muestra una vista en elevación del modelo GL, dimensionado ex profeso para esta investigación. Se trata de un puente en arco con tablero superior de $20 \mathrm{~m}$ de anchura (carretera de cuatro carriles), con una longitud total de $600 \mathrm{~m}$ y un arco con un claro de $400 \mathrm{~m}$ y flecha de $80 \mathrm{~m}$, para una relación flecha/claro de 1/5. El claro y la flecha propuestas para el arco son muy similares a las del actual récord mundial, el Puente Wanxian, en China, que tiene un claro de $420 \mathrm{~m}$. El modelo está compuesto por 10 claros parciales sobre el arco de $40 \mathrm{~m}$ cada uno, cuatro claros de $35 \mathrm{~m}$ (dos a cada lado del arco), y dos claros de $30 \mathrm{~m}$ (uno en cada extremo). En la fig. 22 se presentan las secciones transversales de la estructura. El peralte del arco es linealmente variable en los primeros $60 \mathrm{~m}$, iniciando con $7 \mathrm{~m}$ en los arranques y disminuyendo hasta $5.5 \mathrm{~m}$, continuando con este valor en el resto.

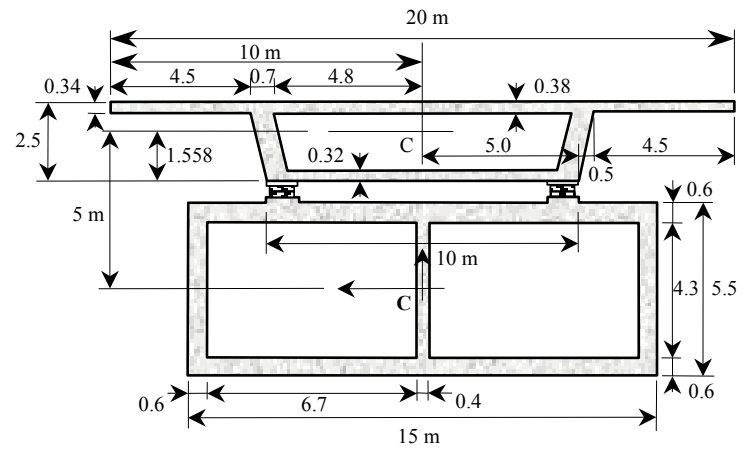

a) Tablero y arco. Corte por el centro del claro

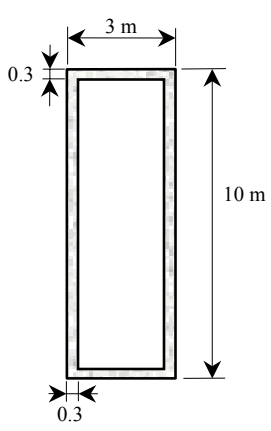

b) Pilas 1, 2, 4 a 12, 14 y 15

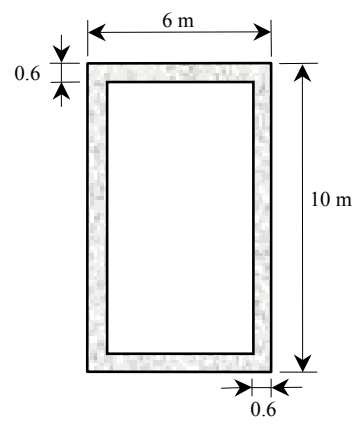

c) Pilas 3 y 13

Figura 22. Secciones transversales del modelo GL

Todas las pilas desplantadas sobre el arco se consideraron continuas con éste. Las dos pilas principales ( $\mathrm{P} 3$ y $\mathrm{P} 13$ ) se consideraron continuas con la superestructura. Las pilas adyacentes a cada lado de las principales (P2, P4, P12 y P14) se supusieron articuladas en su conexión con la superestructura; se decidió sujetarlas en su extremo superior dada su esbeltez. El resto de las pilas, incluyendo la conexión clave del arco - superestructura (P1, P5 a P11, y P15) y ambos estribos, se consideraron equipados en su extremo superior con aparatos de apoyo pot que permiten el deslizamiento longitudinal y el giro de la superestructura sobre la subestructura. Finalmente, se supuso que todas las pilas discontinuas y ambos estribos estarían dotados de llaves 
de cortante que tomasen los desplazamientos transversales de la superestructura. Como consecuencia, despreciando la fricción en los aparatos pot, las pilas P2, P3, P4, P12, P13 y P14 en dirección longitudinal, y todas las pilas, el arco y ambos estribos en direcciones transversal y vertical resistirían las fuerzas de inercia de la superestructura.

La estructura se modeló mediante elementos barra tridimensionales. Los momentos de inercia y las áreas de cortante de las secciones transversales de las pilas, de concreto reforzado, se consideraron iguales al $70 \%$ de la sección bruta, con la finalidad de tomar en cuenta el agrietamiento distribuido a lo largo de ellas; el área y la constante de torsión se tomaron de la sección bruta. Para el arco se consideraron las propiedades de las secciones brutas dado que la elevada fuerza axial de compresión que en él actúa permite despreciar el agrietamiento. Para el tablero, de concreto presforzado, también se consideraron las propiedades de la sección bruta, dado que el agrietamiento en estos elementos es generalmente despreciable. Se consideró un concreto con resistencia a la compresión de $40 \mathrm{MPa}$, con módulo de elasticidad de 31,000 $\mathrm{MPa}$, una relación de Poisson de 0.2 y un peso volumétrico de $24.5 \mathrm{kN} / \mathrm{m}^{3}$.

Los nudos del modelo se ubicaron en cada intersección de elementos y en cada apoyo; además, en las pilas de más de $20 \mathrm{~m}$ de altura se consideraron nudos intermedios con separaciones de entre 10 y $15 \mathrm{~m}$, dado que los modos altos podrían contribuir significativamente a su respuesta dinámica (Priestley et al., 1996, pág. 167). Para el arco se consideraron tres nudos intermedios entre pila y pila, y para el tablero un nudo intermedio en cada tramo, para captar la respuesta en diversos puntos de éstos. En cuanto a las condiciones de apoyo se refiere, las pilas (P1, P2, P3, P13, P14 y P15) y los arranques del arco se consideraron empotrados; se decidió hacer así dado que este tipo de estructuras generalmente se desplantan sobre suelo muy firme o roca, y porque se quería estudiar de manera aislada la respuesta del puente.

\begin{tabular}{|c|c|c|c|c|}
\hline \multicolumn{5}{|c|}{ Tabla 8. Periodos naturales y masa efectiva acumulada para el modelo GL } \\
\hline \multirow{2}{*}{ Modo } & \multirow{2}{*}{ Periodo $(\mathrm{s})$} & \multicolumn{3}{|c|}{ Masa efectiva acumulada (\%) } \\
\cline { 3 - 5 } & & Longitudinal & Transversal & Vertical \\
\hline 1 & 4.340 & 56.3069 & 0.0000 & 0.0000 \\
\hline 2 & 4.288 & 56.3069 & 62.5550 & 0.0000 \\
\hline 3 & 3.161 & 57.6128 & 62.5550 & 0.0000 \\
\hline 4 & 1.790 & 57.6128 & 62.5550 & 0.0000 \\
\hline 5 & 1.690 & 57.6128 & 62.5550 & 0.0001 \\
\hline 6 & 1.227 & 57.6128 & 75.8083 & 0.0001 \\
\hline 7 & 0.967 & 59.7666 & 75.8083 & 0.0003 \\
\hline 8 & 0.904 & 59.7666 & 75.8083 & 0.0003 \\
\hline 9 & 0.808 & 59.7666 & 75.8083 & 43.1109 \\
\hline 10 & 0.637 & 59.7666 & 75.8083 & 61.1145 \\
\hline
\end{tabular}

En la tabla 8 se presentan los periodos naturales y los porcentajes de masa efectiva acumulada para los primeros 10 modos de vibrar de la estructura. Se observa que la masa efectiva acumulada no era suficiente para evaluar adecuadamente la respuesta del modelo, por lo que en los diferentes análisis se consideraron los primeros 75 modos, con lo que la masa efectiva 
acumulada rebasaba ya el $99 \%$ en todos los casos. En la fig. 23 se presentan las configuraciones modales para tres modos de vibrar, que corresponden a los modos longitudinal, transversal y vertical que más contribuyeron a la respuesta.

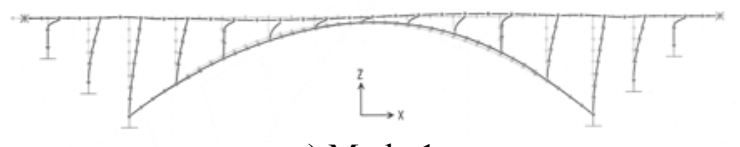

a) Modo 1

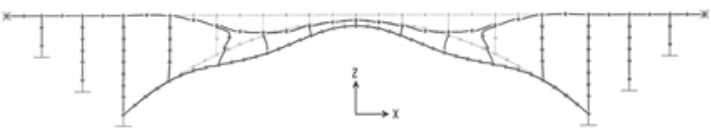

c) Modo 9

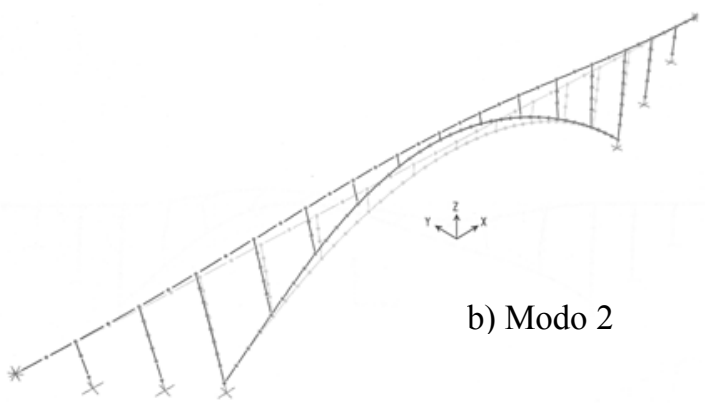

Figura 23. Configuraciones modales del modelo GL

Se evaluó la respuesta sísmica del modelo GL en el plano del puente, sometiéndolo al evento descrito para el Puente Bixby Creek y que incluye efectos de fuente cercana. En la dirección longitudinal se aplicó el acelerograma de la fig. 13 (normal a la falla) y simultáneamente el correspondiente a la dirección vertical (McCallen et al., 1999). El análisis paso a paso con movimiento uniforme de los apoyos se efectuó con ayuda del programa $S A P 2000$ Nonlinear (CSI, 1997). El objetivo del estudio paramétrico fue evaluar el comportamiento del modelo sin dispositivos disipadores de energía y empleando amortiguadores de fluidos viscosos regidos por la ecuación constitutiva (Seleemah y Constantinou, 1997)

$$
F=C \cdot V^{n}
$$

donde $F$ es la fuerza de amortiguamiento, $V$ es la velocidad a través del dispositivo, $C$ es un coeficiente de amortiguamiento y el exponente $n$ define el tipo de amortiguador. Estos dispositivos pueden fabricarse para un amplio intervalo de valores de $C$ y $n$. Para los modelos con amortiguadores se colocó uno en cada extremo del tablero, conectando la superestructura con los estribos, los que se supusieron completamente fijos al terreno. Con la finalidad de obtener los amortiguadores óptimos se consideraron valores del coeficiente $C$ comprendidos entre 1,000 y $100,000 \mathrm{kN}(\mathrm{s} / \mathrm{m})^{n}$; para cada uno de estos coeficientes se consideraron valores del exponente $n$ de $1 / 4,1 / 2$ y 1 . El software empleado permite modelar amortiguadores viscoelásticos tipo Maxwell (un resorte y un amortiguador viscoso conectados en serie) mediante los elementos tipo Nllink. Para todos los casos se supuso un amortiguamiento viscoso de la estructura igual al 5\%.

Se analizaron un total de 22 casos. La respuesta del puente se monitoreó en función del tiempo en varios puntos de interés. Uno de los más significativos fue el desplazamiento longitudinal de la superestructura. En la fig. 24.a) se presenta la historia temporal de desplazamientos para los modelos GL básico y con $C=10,000 \mathrm{kN}(\mathrm{s} / \mathrm{m})^{1 / 4}$. El desplazamiento máximo longitudinal que alcanzó el modelo sin amortiguadores fue de $0.70 \mathrm{~m}$ para un tiempo de 12.64 s. En la misma figura se observa la reducción de la respuesta al colocar los amortiguadores; 
los más eficientes para este caso resultaron los que tienen un exponente $n=1 / 4$, que redujeron el desplazamiento máximo a tan sólo $0.29 \mathrm{~m}$ para un tiempo de $6.62 \mathrm{~s}$.

La fig. 24.b) muestra la fuerza máxima que se generó en cada amortiguador en función del parámetro $C$. Cada punto de esta gráfica proviene de los resultados de un análisis dinámico usando una pareja de valores de $C$ y $n$. La tendencia fue suficientemente clara, pues resultaron mejor los amortiguadores con $n=1 / 4$, ya que para un mismo valor de $C$ pudieron desarrollar una fuerza mayor disminuyendo así la respuesta de la estructura. La fig. 24.c) muestra la deformación máxima en los amortiguadores, que a su vez es el desplazamiento longitudinal del tablero, en función del coeficiente $C$. Nuevamente, los amortiguadores con el valor más pequeño de $n$ acusan un mejor desempeño, ya que para una misma $C$ reducen en mayor medida la deformación máxima. Nótese que para $C>30,000 \mathrm{kN}(\mathrm{s} / \mathrm{m})^{\mathrm{n}}$ la tasa de reducción en la deformación disminuyó. Además, en la fig. 24.d) se presenta la energía disipada acumulada en los amortiguadores en función del parámetro $C$. Una vez más, resultaron más eficientes los amortiguadores con $n=1 / 4$, salvo para los valores mayores de $C$; también para $C>30,000 \mathrm{kN}(\mathrm{s} / \mathrm{m})^{\mathrm{n}}$ el incremento en la energía disipada disminuyó notablemente.

Las fuerzas internas máximas en la base de las pilas 3 y 13, originadas por el evento en cuestión, se presentan en la fig. 24.e). Se aprecia que la fuerza axial por sismo prácticamente no disminuyó, apenas una tendencia del $10 \%$ en el mejor de los casos. Por el contrario, el momento flexionante máximo debido a sismo tuvo un descenso notable, alcanzándose una tendencia a un mínimo para $C=30,000 \mathrm{kN}(\mathrm{s} / \mathrm{m})^{\mathrm{n}}$. El aporte de los amortiguadores en el decremento de los desplazamientos longitudinales del tablero se ve reflejado en la disminución del momento flexionante en las pilas principales, ya que éstas se supusieron continuas con la superestructura, y por lo tanto muy sensibles a cualquier movimiento en su extremo superior. Para la configuración estructural seleccionada, el arco se vio menos favorecido; en la fig. 24.f) se presentan sus respuestas máximas. La fuerza axial debida a sismo casi no varió al incorporar los amortiguadores, y el momento flexionante apenas disminuyó un 15\% en el mejor de los casos.

En resumen, se ha podido identificar los amortiguadores más eficientes para el sismo y el modelo en estudio, que de acuerdo con la evidencia presentada serían amortiguadores con parámetros $C=30,000 \mathrm{kN}(\mathrm{s} / \mathrm{m})^{\mathrm{n}}, n=1 / 4$ y que sean capaces de desarrollar una fuerza máxima de aproximadamente $25,000 \mathrm{kN}$ (fig. 24.b); dicha fuerza puede alcanzarse, por ejemplo, con cinco amortiguadores de 5,000 kN cada uno, disponibles en el mercado.

\section{DISCUSIÓN}

La construcción de puentes arco ha resurgido a nivel mundial gracias al método de avance en voladizo, y aunque estos sistemas pueden constituir una solución competitiva para salvar claros entre los 200 y los 600 m (Muller, 2001), poco se recurre a estos clásicos de la ingeniería de puentes como solución estructural. Los arcos son estructuras que se diseñan para que su directriz coincida con el antifunicular de las cargas gravitatorias, buscando un desempeño eficiente bajo carga axial de compresión, con flexiones nulas o muy pequeñas. Obviamente, las acciones sísmicas generan condiciones cambiantes que modifican su respuesta estructural. 


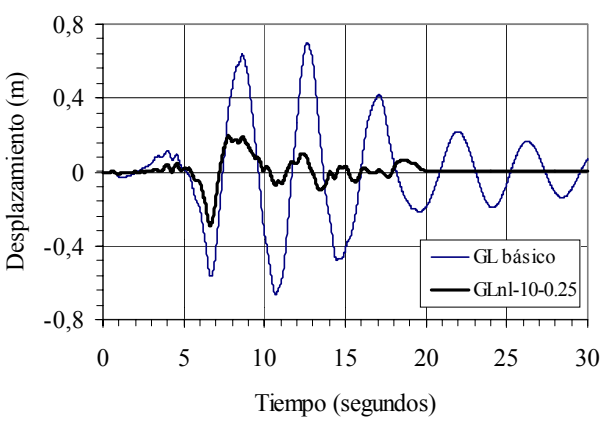

a) Desplazamiento longitudinal del tablero

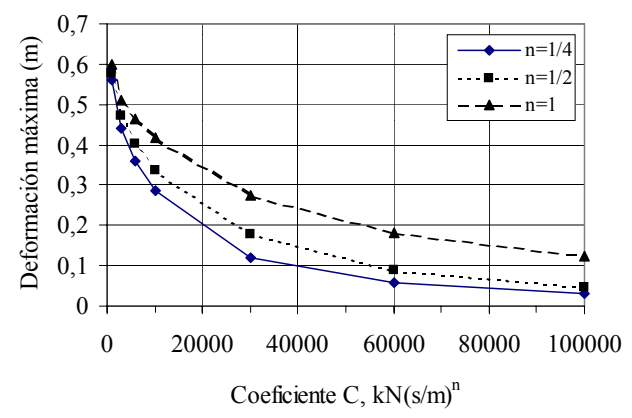

c) Deformación máxima en los amortiguadores

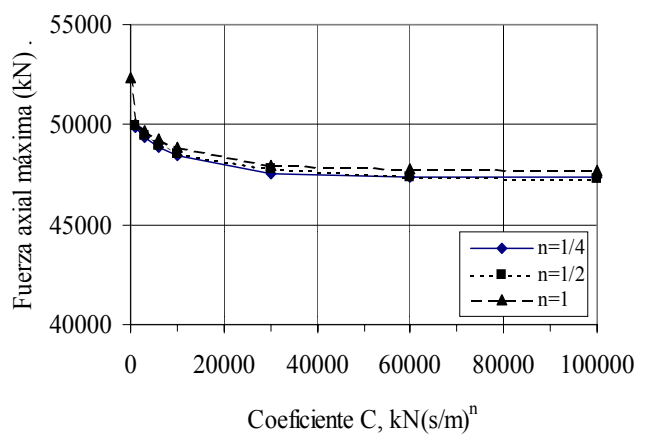

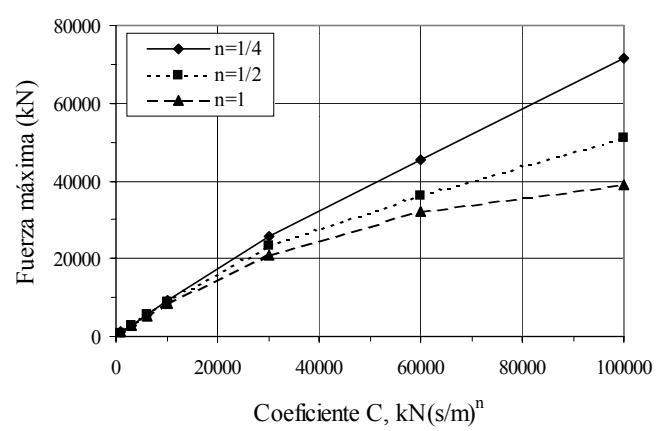

b) Fuerza máxima en los amortiguadores

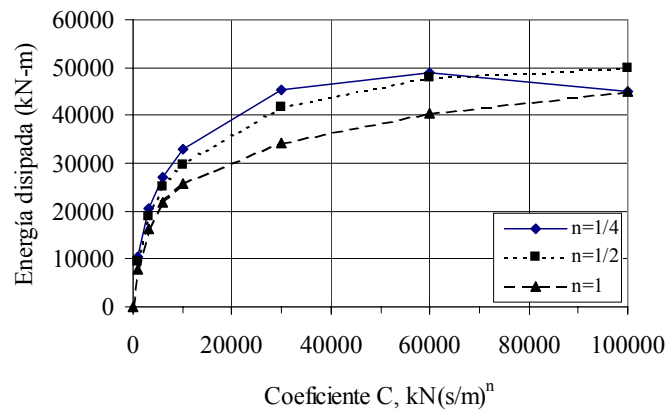

d) Energía disipada por los amortiguadores

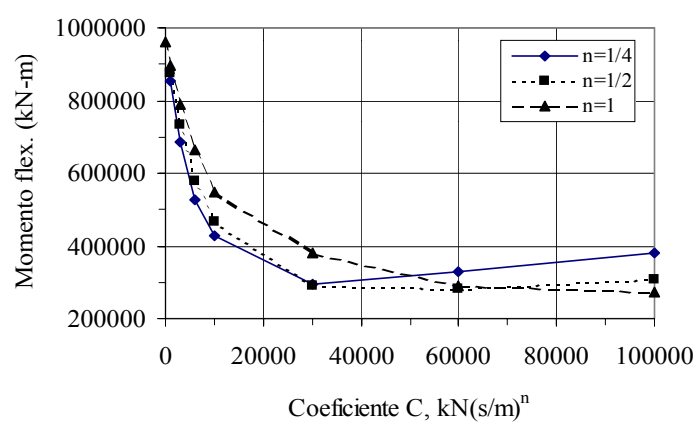

e) Fuerzas internas máximas por sismo en la base de las pilas 3 y 13
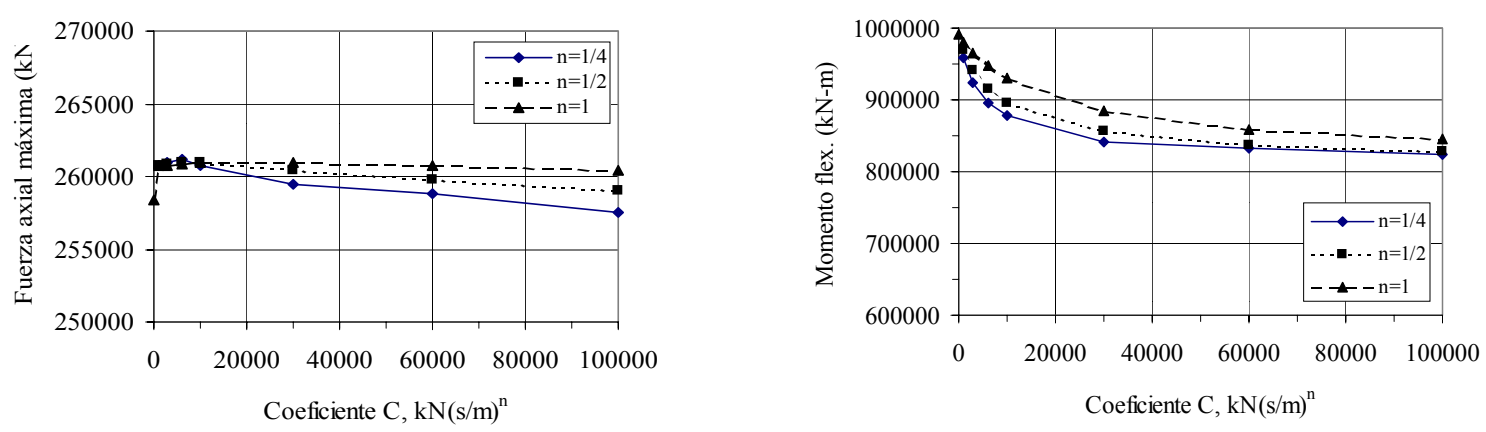

f) Fuerzas internas máximas por sismo en los arranques del arco

Figura 24. Respuesta sísmica del modelo GL ante un evento de fuente cercana 
Los puentes arco son estructuras que exhiben un comportamiento complejo durante la ocurrencia de movimientos sísmicos fuertes (Japan Road Association, 1996), y para evaluar su respuesta de manera objetiva debe recurrirse a análisis dinámicos inelásticos que consideren la naturaleza espacial del movimiento, incluyendo los modos superiores de vibrar de la estructura que sean necesarios, pues éstos pueden tener una participación muy importante en la predicción de la respuesta. El uso de métodos estáticos empleando un coeficiente sísmico, como se hacía anteriormente en Japón (Kawashima y Mizoguti, 2000), han quedado en desuso; inclusive el análisis pushover, usado para evaluar el comportamiento estructural y las demandas sísmicas de otras tipologías de puentes, no puede reproducir fielmente la respuesta dinámica (Krawinkler y Seneviratna, 1998). Las acciones sísmicas en los estados elástico e inelástico generan condiciones cambiantes de los elementos mecánicos, de los desplazamientos, posible fluencia de los apoyos y efectos adicionales de pandeo dentro o fuera del plano. La presencia de la compresión mantiene latentes los problemas no lineales de pandeo modificándose las cargas críticas de diseño, mismas que dependen de las condiciones de apoyo, del comportamiento de los materiales, de las propiedades de las secciones transversales, de la longitud del eje y del claro del arco, de las características del tablero, de la vinculación arco-tablero, y de las posibilidades de movimiento lateral.

Para el cálculo de las propiedades dinámicas de estos puentes es necesario tomar en cuenta el efecto de la gran carga axial que actúa en el arco, y que contribuye a disminuir la rigidez del sistema, inclusive puede volverlo inestable. Además, durante la ocurrencia de un movimiento sísmico fuerte, se presenta una variación muy importante de la fuerza axial en el arco, por lo que es necesario considerar la interacción no lineal de dicha fuerza axial y el momento flexionante. Existen tres métodos que pueden ser aplicados para considerar la fluctuación de la fuerza axial (Nakagawa et al., 2000):

1. Las relaciones momento-curvatura se modelan para cambiar de acuerdo con el valor de la fuerza axial,

2. El efecto de la fluctuación de la fuerza axial es considerado directamente en el análisis empleando elementos fibra, y

3. Inicialmente, se emplea la relación momento-curvatura debida a la fuerza axial causada por la carga muerta y se hace un primer análisis. En seguida se reanaliza la estructura con las relaciones momento-curvatura debidas a la fuerza axial mínima y máxima ocasionadas por la carga gravitacional más sismo, para estudiar de manera aproximada el efecto de la variación de la fuerza axial.

Este fenómeno de fluctuación de la carga axial durante el movimiento sísmico ha sido considerado en algunos estudios relativos a puentes de acero (Nazmy y Konidaris, 1994; Nazmy, 1996), pero se nota la falta de un modelo confiable para el caso de concreto reforzado (Kawashima y Mizoguti, 2000; Nakagawa et al., 2000). Se requiere mayor investigación al respecto.

Los puentes arco de gran claro son sistemas muy flexibles, con periodos largos. Para evaluar su respuesta deben incluirse pulsos de desplazamiento de fuente cercana si la estructura se localiza en las proximidades de una falla activa, como lo considera el reglamento japonés 
(Japan Road Association, 1996). Además, es necesario tomar en cuenta el movimiento no sincrónico del suelo en los desplantes de la cimentación, fenómeno conocido como variabilidad espacial, y que es causado por la diferencia en tiempos de llegada de las ondas sísmicas a los diferentes apoyos, la pérdida de coherencia del movimiento y la influencia de las condiciones locales del suelo (Der Kiureghian y Neuenhofer, 1992). Al respecto, el Eurocódigo 8 Parte 2 (Comité Europeo de Normalización, 1998), recomienda tomar en cuenta este efecto, que afecta principalmente la respuesta de los puentes en sentido longitudinal, si existen discontinuidades geológicas o características topográficas capaces de introducir variaciones importantes en las características del movimiento del suelo o si la longitud del puente es mayor que $600 \mathrm{~m}$; la evidencia presentada por Nazmy y Konidaris (1994) sugiere que este efecto es importante ya para claros de $400 \mathrm{~m}$.

El diseño estructural del arco es inherentemente no dúctil, por lo que los niveles de fuerza sísmica debieran estar basados en una respuesta elástica (Priestley et al., 1996, pág. 154). Hasta ahora se ha acumulado poca experiencia en desastres que tengan que ver con puentes colgantes, atirantados o en arco, y los métodos de evaluación de la ductilidad de sus elementos no han sido valorados (Japan Road Association, 1996). El Eurocódigo 8 Parte 2 (Comité Europeo de Normalización, 1998), señala que para puentes especiales, como los arcos, es preferible seleccionar un comportamiento sísmico elástico. Si se quiere mantener los elementos estructurales de los puentes arco con una respuesta elástica y a la vez contar con una estructura capaz de disipar energía durante la ocurrencia de movimientos sísmicos, se puede recurrir al uso de dispositivos disipadores. Hasta ahora no ha sido evaluada la respuesta sísmica de estas estructuras empleando sistemas de control, por lo que los autores están realizando una investigación en la que se evalúa la respuesta sísmica de puentes arco equipados con amortiguadores viscosos; los primeros resultados han sido presentados al final de la parte referente a puentes de concreto.

Hasta ahora sólo se han evaluado modelos considerando la estructura empotrada en la cimentación. Sin embargo, el comportamiento de las cimentaciones desplantadas superficialmente en roca o suelo firme, o el de cimentaciones profundas, modifica los empujes laterales en los arranques del arco, cambiando su estado de flexión, con una consecuente redistribución de las fuerzas y de las relaciones de interacción fuerza axial-momento. Adicionalmente, del comportamiento sísmico de los arcos durante la construcción no se tienen antecedentes.

\section{COMENTARIOS FINALES}

Los puentes son obras civiles de las que se requiere su integridad estructural y accesibilidad después de la ocurrencia de un sismo. Sin embargo, algunos movimientos telúricos fuertes recientes han mostrado que los puentes, en general, son estructuras sísmicamente vulnerables, aunque hasta ahora los puentes arco no han sufrido daño severo por sismo. En aras de contribuir a comprender mejor la interacción de las variables que influyen en el comportamiento de estas estructuras, se ha tratado de mostrar un panorama del estado del conocimiento sobre la respuesta sísmica de puentes arco, sistemas que exhiben un comportamiento complejo durante la ocurrencia 
de movimientos sísmicos fuertes, y que para ser evaluadas requieren de análisis dinámicos tridimensionales no lineales. Aspectos como la interacción suelo estructura, la variabilidad espacial, los efectos de fuente cercana, la interacción fuerza axial-momento y la respuesta empleando sistemas de control requieren de un mayor desarrollo.

\section{REFERENCIAS}

Alvarez, J J, N Parra y A Aparicio (2002), "Comportamiento estructural de puentes en arco sometidos a movimiento sísmico de fuente cercana", Memorias, XIII Congreso Nacional de Ingeniería Estructural, Puebla, México, CDROM, Artículo No. 158.

Billington, D P (1977), "History and esthetics in concrete arch bridges", ASCE Journal of the Structural Division, Vol. 103, No. ST11, pp. 2129-2143.

Boitel, P y C De La Fuente (1998), “Aseismic devices for bridges", Memorias, Thirteen FIP Congress, Amsterdam, pp. 105-109.

Comité Europeo de Normalización (1998), Eurocódigo 8: disposiciones para el proyecto de estructuras sismorresistentes. Parte 2: puentes, ENV 1998-2, Asociación Española de Certificación y Normalización, 90 p.

Computers and Structures, Inc. (1997), "SAP2000, integrated finite element analysis and design of structures”, Structural Analysis Program, NonLinear Version, Berkeley, California.

Der Kiureghian, A y A Neuenhofer (1992), "Response spectrum method for multi-support seismic excitations", Earthquake Engineering and Structural Dynamics, Vol. 21, pp. 713-740.

Dusseau, R A y R K Wen (1989), “Seismic responses of deck-type arch bridges”, Earthquake Engineering and Structural Dynamics, Vol. 18, pp. 701-715.

Horinouchi, S, T Sakata y K Uno (1996), "Analysis of fixed reinforced concrete (RC) arch bridge with small section members under earthquakes", Memorias, $11^{\text {th }}$ World Conference on Earthquake Engineering, Acapulco, México, CDROM, Artículo No. 1305.

Japan Road Association (1996), Specifications for highway bridges. Part V: seismic design, Tokio, $207 \mathrm{p}$.

Kawashima, K (2000), "Seismic design and retrofit of bridges", Memorias, $12^{\text {th }}$ World Conference on Earthquake Engineering, Auckland, Nueva Zelanda, CDROM, Artículo No. 2828.

Kawashima, K y A Mizoguti (2000), "Seismic response of a reinforced concrete arch bridge", Memorias, $12^{\text {th }}$ World Conference on Earthquake Engineering, Auckland, Nueva Zelanda, CDROM, Artículo No. 1824.

Krawinkler, H y G D P K Seneviratna (1998), "Pros and cons of a pushover analysis for seismic performance evaluation”, Journal of Engineering Structures, Vol. 20, No. 4-6, pp. 452-464.

Kuranishi, S y A Nakajima (1986), "Strength characteristics of steel arch bridges subjected to longitudinal acceleration”, JSCE Structural Engineering/Earthquake Engineering, Vol. 3, No. 2, pp. 287-295. 
Lee, C-M (1990), "Nonlinear seismic analysis of steel arch bridges", Tesis Doctoral, Michigan State University, $216 \mathrm{p}$.

Lobo, W (2000), "Diseño sísmico de puentes: estado del arte", Revista de Ingeniería Sísmica, SMIS, No. 63, pp. 55-71.

Manterola, J (1999), "Técnicas avanzadas de diseño y construcción de puentes", Tomo III, Seminario de la Asociación Mexicana de Ingeniería de Vías Terrestres, A. C., México.

McCallen, D, C Noble y M Hoehler (1999), "The seismic response of concrete arch bridges: with focus on the Bixby Creek Bridge, Carmel, California", Report No. UCRL-ID-134419, Lawrence Livermore National Laboratory, California, 170 p.

Muller, J (2001), "On design and construction of long span concrete arch bridges", Memorias, Third International Arch Bridges Conference ARCH'01,París, Francia, pp. 17-26.

Nakagawa, K, T Irie, A D Sumaya y K Oda (2000), "Seismic design of arch bridges during strong earthquake", Memorias, $12^{\text {th }}$ World Conference on Earthquake Engineering, Auckland, Nueva Zelanda, CDROM, Artículo No. 1926.

Nazmy, A S (1996), "Earthquake-response characteristics of long-span arch bridges", Memorias, $11^{\text {th }}$ World Conference on Earthquake Engineering, Acapulco, México, CDROM, Artículo No. 1309.

Nazmy, A S y E G Konidaris (1994), "Nonlinear seismic behavior of steel deck-type arch bridges", Memorias, $5^{\text {th }}$ US National Conference on Earthquake Engineering, Chicago, E. U. A., pp. 367-376,

Priestley, M J N, F Seible y G M Calvi (1996), Seismic Design and Retrofit of Bridges, John Wiley and Sons, Nueva York, $686 \mathrm{p}$.

Seleemah, A A y M C Constantinou (1997), "Investigation of Seismic Response of Buildings with Linear and Nonlinear Fluid Viscous Dampers", Technical Report NCEER-97-0004, National Center for Earthquake Engineering, Buffalo, Nueva York, 262 p.

Simunic, Z, B Pavlovic y J Bleiziffer (2001), "Dynamic behaviour of concrete arch bridges on the Adriatic coast", Memorias, Third International Arch Bridges Conference ARCH'01, París, Francia, pp. 641-646.

Spielmann, A (2001), "An arch of 600 meters", Memorias, Third International Arch Bridges Conference ARCH'01, París, Francia, pp. 683-692. 